\title{
G2HDM: Gauged Two Higgs Doublet Model
}

\author{
Wei-Chih Huang, ${ }^{a, b}$ Yue-Lin Sming Tsai ${ }^{c}$ and Tzu-Chiang Yuan ${ }^{d, e}$ \\ ${ }^{a}$ Department of Physics and Astronomy, University College London, \\ London, U.K. \\ ${ }^{b}$ Fakultät für Physik, Technische Universität Dortmund, \\ 44221 Dortmund, Germany \\ ${ }^{c}$ Kavli IPMU (WPI), University of Tokyo, \\ Kashiwa, Chiba 277-8583, Japan \\ ${ }^{d}$ Institute of Physics, Academia Sinica, \\ Nangang, Taipei 11529, Taiwan \\ ${ }^{e}$ Physics Division, National Center for Theoretical Sciences, \\ Hsinchu, Taiwan \\ E-mail: wei-chih.huang@tu-dortmund.de, yue-lin.tsai@ipmu.jp, \\ tcyuan@phys.sinica.edu.tw
}

ABstract: A novel model embedding the two Higgs doublets in the popular two Higgs doublet models into a doublet of a non-abelian gauge group $\mathrm{SU}(2)_{H}$ is presented. The Standard Model $\mathrm{SU}(2)_{L}$ right-handed fermion singlets are paired up with new heavy fermions to form $\mathrm{SU}(2)_{H}$ doublets, while $\mathrm{SU}(2)_{L}$ left-handed fermion doublets are singlets under $\mathrm{SU}(2)_{H}$. Distinctive features of this anomaly-free model are: (1) Electroweak symmetry breaking is induced from spontaneous symmetry breaking of $\mathrm{SU}(2)_{H}$ via its triplet vacuum expectation value; (2) One of the Higgs doublet can be inert, with its neutral component being a dark matter candidate as protected by the $\mathrm{SU}(2)_{H}$ gauge symmetry instead of a discrete $Z_{2}$ symmetry in the usual case; (3) Unlike Left-Right Symmetric Models, the complex gauge fields $\left(W_{1}^{\prime} \mp i W_{2}^{\prime}\right)$ (along with other complex scalar fields) associated with the $\mathrm{SU}(2)_{H}$ do not carry electric charges, while the third component $W_{3}^{\prime}$ can mix with the hypercharge $\mathrm{U}(1)_{Y}$ gauge field and the third component of $\mathrm{SU}(2)_{L}$; (4) Absence of tree level flavour changing neutral current is guaranteed by gauge symmetry; and etc. In this work, we concentrate on the mass spectra of scalar and gauge bosons in the model. Constraints from previous $Z^{\prime}$ data at LEP and the Large Hadron Collider measurements of the Standard Model Higgs mass, its partial widths of $\gamma \gamma$ and $Z \gamma$ modes are discussed.

Keywords: Beyond Standard Model, Higgs Physics

ArXiv EPrint: 1512.00229 


\section{Contents}

1 Introduction 1

2 G2HDM set up $\quad 4$

2.1 Higgs potential 4

2.2 Yukawa couplings 6

$\begin{array}{ll}2.3 \text { Anomaly cancellation } & 7\end{array}$

3 Spontaneous symmetry breaking and mass spectra $\quad 8$

$\begin{array}{lll}3.1 & \text { Spontaneous symmetry breaking } & 9\end{array}$

3.2 Scalar mass spectrum $\quad 9$

$\begin{array}{lll}3.3 & \mathrm{SU}(2)_{H} \times \mathrm{U}(1)_{X} \text { gauge boson mass spectrum } & 11\end{array}$

4 Phenomenology 14

4.1 Numerical solutions for scalar and gauge boson masses 14

$\begin{array}{lll}4.2 Z^{\prime} \text { constraints } & 17\end{array}$

4.3 Constraints from the $125 \mathrm{GeV}$ SM-like Higgs 19

$\begin{array}{lll}4.4 & \text { Dark matter stability } & 21\end{array}$

4.5 Dark matter relic density 22

4.6 EWPT $-\Delta S, \Delta T$ and $\Delta U \quad 24$

5 Conclusions and outlook $\quad 25$

A Analytical expression for $v, v_{\Delta}$ and $v_{\Phi} \quad 26$

$\begin{array}{ll}\text { B Decay width of SM Higgs to } \gamma \gamma \text { and } Z \gamma & 27\end{array}$

\section{Introduction}

Despite the historical discovery of the $125 \mathrm{GeV}$ scalar resonance at the LHC [1,2] completes the last building block of the Standard Model (SM), many important questions are still lingering in our minds. Is this really the Higgs boson predicted by the SM or its imposter? Are there any other scalars hidden somewhere? What is the dark matter (DM) particle? And so on. Extended Higgs sector is commonly used to address various theoretical issues such as DM. The simplest solution for DM is to add a gauge-singlet scalar, odd under a $Z_{2}$ symmetry [3-5]. In the context of supersymmetric theories like the Minimal Supersymmetric Standard Model (MSSM) proposed to solve the gauge hierarchy problem, an additional second Higgs doublet is mandatory due to the requirement of anomaly cancelation and supersymmetry (SUSY) chiral structure. In the inert two Higgs doublet model (IHDM) [6], since the second Higgs doublet is odd under a $Z_{2}$ symmetry its neutral component can be 
a DM candidate [7-10]. Moreover the observed baryon asymmetry cannot be accounted for in the SM since the CKM phase in the quark sector is too small to generate sufficient baryon asymmetry [11-13], and the Higgs potential cannot achieve the strong first-order electroweak phase transition unless the SM Higgs boson is lighter than $70 \mathrm{GeV}[14,15]$. A general two Higgs doublet model $(2 \mathrm{HDM})$ contains additional $C P$-violation source [16-20] in the scalar sector and hence it may circumvent the above shortcomings of the SM for the baryon asymmetry.

The complication associated with a general 2HDM [21] stems from the fact that there exist many terms in the Higgs potential allowed by the SM gauge symmetry, including various mixing terms between two Higgs doublets $H_{1}$ and $H_{2}$. In the case of both doublets develop vacuum expectation values (vevs), the observed 125 Higgs boson in general would be a linear combination of three neutral scalars (or of two $C P$-even neutral scalars if the Higgs potential preserves $C P$ symmetry), resulting in flavour-changing neutral current (FCNC) at tree-level which is tightly constrained by experiments. This Higgs mixing effects lead to changes on the Higgs decay branching ratios into SM fermions which must be confronted by the Large Hadron Collider (LHC) data.

One can reduce complexity in the 2HDM Higgs potential by imposing certain symmetry. The popular choice is a discrete symmetry, such as $Z_{2}$ on the second Higgs doublet in IHDM or 2HDM type-I [22, 23], type-II [23, 24], type-X and type-Y [25-27] where some of SM fermions are also odd under $Z_{2}$ unlike IHDM. The other choice is a continuous symmetry, such as a local U(1) symmetry discussed in [28-31]. The FCNC constraints can be avoided by satisfying the alignment condition of the Yukawa couplings [32] to eliminating dangerous tree-level contributions although it is not radiatively stable [33]. Alternatively, the aforementioned $Z_{2}$ symmetry can be used to evade FCNC at tree-level since SM fermions of the same quantum number only couple to one of two Higgs doublets [34, 35]. Moreover, the Higgs decay branching ratios remain intact in the IHDM since $H_{1}$ is the only doublet which obtains the vacuum expectation value (vev), or one can simply make the second Higgs doublet $H_{2}$ much heavier than the SM one such that $H_{2}$ essentially decouples from the theory. All in all, the IHDM has many merits, including accommodating DM, avoiding stringent collider constraints and having a simpler scalar potential. The required $Z_{2}$ symmetry, however, is just imposed by hand without justification. The lack of explanation prompts us to come up with a novel 2HDM, which has the same merits of IHDM with an simpler two-doublet Higgs potential but naturally achieve that $H_{2}$ does not obtain a vev, which is reinforced in IHDM by the artificial $Z_{2}$ symmetry.

In this work, we propose a $2 \mathrm{HDM}$ with additional $\mathrm{SU}(2)_{H} \times \mathrm{U}(1)_{X}$ gauge symmetry, where $H_{1}$ (identified as the SM Higgs doublet) and $H_{2}$ form an $\mathrm{SU}(2)_{H}$ doublet such that the two-doublet potential itself is as simple as the SM Higgs potential with just a quadratic mass term plus a quartic term. The price to pay is to introduce additional scalars: one $\mathrm{SU}(2)_{H}$ triplet and one $\mathrm{SU}(2)_{H}$ doublet (which are all singlets under the SM gauge groups) with their vevs providing masses to the new gauge bosons. At the same time, the vev of the triplet induces the SM Higgs vev, breaking $\mathrm{SU}(2)_{L} \times \mathrm{U}(1)_{Y}$ down to $\mathrm{U}(1)_{Q}$, while $H_{2}$ do not develop any vev and the neutral component of $H_{2}$ could be a DM candidate, whose stability is protected by the $\mathrm{SU}(2)_{H}$ gauge symmetry and Lorentz invariance. In order 
to write down $\mathrm{SU}(2)_{H} \times \mathrm{U}(1)_{X}$ invariant Yukawa couplings, we introduce heavy $\mathrm{SU}(2)_{L}$ singlet Dirac fermions, the right-handed component of which is paired up with the SM right-handed fermions to comprise $\mathrm{SU}(2)_{H}$ doublets. The masses of the heavy fermions come from the vev of the $\mathrm{SU}(2)_{H}$ doublet. In this setup, the model is anomaly-free with respect to all gauge groups. In what follows, we will abbreviate our model as G2HDM, the acronym of gauged 2 Higgs doublet model.

We here concentrate on the scalar and additional gauge mass spectra of G2HDM and various collider constraints. DM phenomenology will be addressed in a separate publication. As stated before, the neutral component of $H_{2}$ or any neutral heavy fermion inside an $\mathrm{SU}(2)_{H}$ doublet can potentially play a role of stable $\mathrm{DM}$ due to the $\mathrm{SU}(2)_{H}$ gauge symmetry without resorting to an ad-hoc $Z_{2}$ symmetry. It is worthwhile to point out that the way of embedding $H_{1}$ and $H_{2}$ into the $\mathrm{SU}(2)_{H}$ doublet is similar to the Higgs bi-doublet in the Left-Right Symmetric Model (LRSM) [36-40] based on the gauge group $\mathrm{SU}(2)_{L} \times \mathrm{SU}(2)_{R} \times \mathrm{U}(1)_{B-L}$, where $\mathrm{SU}(2)_{L}$ gauge bosons connect fields within $H_{1}$ or $H_{2}^{*}$, whereas the heavy $\mathrm{SU}(2)_{R}$ gauge bosons transform $H_{1}$ into $H_{2}^{*}$. The main differences between G2HDM and LRSM are:

- The charge assignment on the $\mathrm{SU}(2)_{H}$ Higgs doublet is charged under $\mathrm{U}(1)_{Y} \times \mathrm{U}(1)_{X}$ while the bi-doublet in LRSM is neutral under $\mathrm{U}(1)_{B-L}$. Thus the corresponding Higgs potential are much simpler in G2HDM;

- All $\mathrm{SU}(2)_{H}$ gauge bosons are electrically neutral whereas the $W_{R}^{ \pm}$of $\mathrm{SU}(2)_{R}$ carry electric charge of one unit;

- The SM right-handed fermions such as $u_{R}$ and $d_{R}$ do not form a doublet under $\mathrm{SU}(2)_{H}$ unlike in LRSM where they form a $\mathrm{SU}(2)_{R}$ doublet, leading to very different phenomenology.

On the other hand, this model is also distinctive from the twin Higgs model [41, 42] where $H_{1}$ and $H_{2}$ are charged under two different gauge groups $\mathrm{SU}(2)_{A}$ (identified as the SM gauge group $\left.\mathrm{SU}(2)_{L}\right)$ and $\mathrm{SU}(2)_{B}$ respectively, and the mirror symmetry on $\mathrm{SU}(2)_{A}$ and $\mathrm{SU}(2)_{B}$, i.e., $g_{A}=g_{B}$, can be used to cancel quadratic divergence of radiative corrections to the SM Higgs mass. Solving the little hierarchy problem is the main purpose of twin Higgs model while G2HDM focuses on getting an inert Higgs doublet as the DM candidate without imposing an ad-hoc $Z_{2}$ symmetry. Finally, embedding two Higgs doublets into a doublet of a non-abelian gauge symmetry with electrically neutral bosons has been proposed in refs. [43-45], where the non-abelian gauge symmetry is called $\mathrm{SU}(2)_{N}$ instead of $\mathrm{SU}(2)_{H}$. Due to the $E_{6}$ origin, those models have, nonetheless, quite different particle contents for both fermions and scalars as well as varying embedding of SM fermions into $\mathrm{SU}(2)_{N}$, resulting in distinct phenomenology from our model.

This paper is organized as follows. In section 2, we specify the model. We discuss the complete Higgs potential, Yukawa couplings for SM fermions as well as new heavy fermions and anomaly cancellation. In section 3, we study spontaneous symmetry breaking conditions (section 3.1), and analyze the scalar boson mass spectrum (section 3.2) and the extra gauge boson mass spectrum (section 3.3). We discuss some phenomenology 


\begin{tabular}{|c|c|c|c|c|c|}
\hline Matter Fields & $\mathrm{SU}(3)_{C}$ & $\mathrm{SU}(2)_{L}$ & $\mathrm{SU}(2)_{H}$ & $\mathrm{U}(1)_{Y}$ & $\mathrm{U}(1)_{X}$ \\
\hline$Q_{L}=\left(\begin{array}{ll}u_{L} & d_{L}\end{array}\right)^{T}$ & 3 & 2 & 1 & $1 / 6$ & 0 \\
\hline$U_{R}=\left(\begin{array}{ll}u_{R} & u_{R}^{H}\end{array}\right)^{T}$ & 3 & 1 & 2 & $2 / 3$ & 1 \\
\hline$D_{R}=\left(\begin{array}{ll}d_{R}^{H} & d_{R}\end{array}\right)^{T}$ & 3 & 1 & 2 & $-1 / 3$ & -1 \\
\hline$L_{L}=\left(\begin{array}{ll}\nu_{L} & e_{L}\end{array}\right)^{T}$ & 1 & 2 & 1 & $-1 / 2$ & 0 \\
\hline$N_{R}=\left(\begin{array}{ll}\nu_{R} & \nu_{R}^{H}\end{array}\right)^{T}$ & 1 & 1 & 2 & 0 & 1 \\
\hline$E_{R}=\left(\begin{array}{ll}e_{R}^{H} & e_{R}\end{array}\right)^{T}$ & 1 & 1 & 2 & -1 & -1 \\
\hline$\chi_{u}$ & 3 & 1 & 1 & $2 / 3$ & 0 \\
\hline$\chi_{d}$ & 3 & 1 & 1 & $-1 / 3$ & 0 \\
\hline$\chi_{\nu}$ & 1 & 1 & 1 & 0 & 0 \\
\hline$\chi_{e}$ & 1 & 1 & 1 & -1 & 0 \\
\hline$H=\left(\begin{array}{ll}H_{1} & H_{2}\end{array}\right)^{T}$ & 1 & 2 & 2 & $1 / 2$ & 1 \\
\hline$\Delta_{H}=\left(\begin{array}{cc}\Delta_{3} / 2 & \Delta_{p} / \sqrt{2} \\
\Delta_{m} / \sqrt{2} & -\Delta_{3} / 2\end{array}\right)$ & 1 & 1 & 3 & 0 & 0 \\
\hline$\Phi_{H}=\left(\begin{array}{ll}\Phi_{1} & \Phi_{2}\end{array}\right)^{T}$ & 1 & 1 & 2 & 0 & 1 \\
\hline
\end{tabular}

Table 1. Matter field contents and their quantum number assignments in G2HDM.

in section 4, including numerical solutions for the scalar and gauge bosons masses, $Z^{\prime}$ constraints, SM Higgs decays into the $\gamma \gamma$ and $\gamma Z$, and stability of DM candidate. Finally, we summarize and conclude in section 5 . Useful formulas are relegated to two appendixes.

\section{G2HDM set up}

In this section, we first set up the G2HDM by specifying its particle content and write down the Higgs potential, including the two Higgs doublets $H_{1}$ and $H_{2}$, an $\mathrm{SU}(2)_{H}$ triplet $\Delta_{H}$ and an $\mathrm{SU}(2)_{H}$ doublet $\Phi_{H}$, where $\Delta_{H}$ and $\Phi_{H}$ are singlets under the SM gauge group. Second, we spell out the fermion sector, requiring the Yukawa couplings to obey the $\mathrm{SU}(2)_{H} \times \mathrm{U}(1)_{X}$ symmetry. The abelian $\mathrm{U}(1)_{X}$ factor can be treated as either local or global symmetry as will be elaborated further in later section. There are different ways of introducing new heavy fermions but we choose a simplest realization: the heavy fermions together with the $\mathrm{SM}$ right-handed fermions comprise $\mathrm{SU}(2)_{H}$ doublets, while the SM left-handed doublets are singlets under $\mathrm{SU}(2)_{H}$. We note that heavy right-handed neutrinos paired up with a mirror charged leptons forming $\mathrm{SU}(2)_{L}$ doublets was suggested before in [46]. The matter content of the G2HDM is summarized in table 1. Third, we demonstrate the model is anomaly free.

\subsection{Higgs potential}

We have two Higgs doublets, $H_{1}$ and $H_{2}$ where $H_{1}$ is identified as the SM Higgs doublet and $H_{2}$ (with the same hypercharge $Y=1 / 2$ as $H_{1}$ ) is the additional $\mathrm{SU}(2)_{L}$ doublet. In 
addition to the SM gauge groups, we introduce additional groups, $\mathrm{SU}(2)_{H} \times \mathrm{U}(1)_{X}$ under which $H_{1}$ and $H_{2}$ transform as a doublet, $H=\left(H_{1} H_{2}\right)^{T}$ with $\mathrm{U}(1)_{X}$ charge $X(H)=1$.

With additional $\mathrm{SU}(2)_{H}$ triplet and doublet, $\Delta_{H}$ and $\Phi_{H}$, which are singlets under $\mathrm{SU}(2)_{L}$, the Higgs potential invariant under both $\mathrm{SU}(2)_{L} \times \mathrm{U}(1)_{Y}$ and $\mathrm{SU}(2)_{H} \times$ $\mathrm{U}(1)_{X}$ reads $^{1}$

$$
V\left(H, \Delta_{H}, \Phi_{H}\right)=V(H)+V\left(\Phi_{H}\right)+V\left(\Delta_{H}\right)+V_{\text {mix }}\left(H, \Delta_{H}, \Phi_{H}\right),
$$

with

$$
\begin{aligned}
V(H) & =\mu_{H}^{2} H^{\dagger} H+\lambda_{H}\left(H^{\dagger} H\right)^{2}, \\
& =\mu_{H}^{2}\left(H_{1}^{\dagger} H_{1}+H_{2}^{\dagger} H_{2}\right)+\lambda_{H}\left(H_{1}^{\dagger} H_{1}+H_{2}^{\dagger} H_{2}\right)^{2},
\end{aligned}
$$

which contains just two terms ( 1 mass term and 1 quartic term) as compared to 8 terms (3 mass terms and 5 quartic terms) in general 2HDM [21];

$$
\begin{aligned}
V\left(\Phi_{H}\right) & =\mu_{\Phi}^{2} \Phi_{H}^{\dagger} \Phi_{H}+\lambda_{\Phi}\left(\Phi_{H}^{\dagger} \Phi_{H}\right)^{2}, \\
& =\mu_{\Phi}^{2}\left(\Phi_{1}^{*} \Phi_{1}+\Phi_{2}^{*} \Phi_{2}\right)+\lambda_{\Phi}\left(\Phi_{1}^{*} \Phi_{1}+\Phi_{2}^{*} \Phi_{2}\right)^{2}, \\
V\left(\Delta_{H}\right) & =-\mu_{\Delta}^{2} \operatorname{Tr}\left(\Delta_{H}^{\dagger} \Delta_{H}\right)+\lambda_{\Delta}\left(\operatorname{Tr}\left(\Delta_{H}^{\dagger} \Delta_{H}\right)\right)^{2}, \\
& =-\mu_{\Delta}^{2}\left(\frac{1}{2} \Delta_{3}^{2}+\Delta_{p} \Delta_{m}\right)+\lambda_{\Delta}\left(\frac{1}{2} \Delta_{3}^{2}+\Delta_{p} \Delta_{m}\right)^{2},
\end{aligned}
$$

and finally the mixed term

$$
\begin{aligned}
V_{\text {mix }}\left(H, \Delta_{H}, \Phi_{H}\right)= & +M_{H \Delta}\left(H^{\dagger} \Delta_{H} H\right)-M_{\Phi \Delta}\left(\Phi_{H}^{\dagger} \Delta_{H} \Phi_{H}\right) \\
& +\lambda_{H \Delta}\left(H^{\dagger} H\right) \operatorname{Tr}\left(\Delta_{H}^{\dagger} \Delta_{H}\right)+\lambda_{H \Phi}\left(H^{\dagger} H\right)\left(\Phi_{H}^{\dagger} \Phi_{H}\right) \\
& +\lambda_{\Phi \Delta}\left(\Phi_{H}^{\dagger} \Phi_{H}\right) \operatorname{Tr}\left(\Delta_{H}^{\dagger} \Delta_{H}\right) \\
= & +M_{H \Delta}\left(\frac{1}{\sqrt{2}} H_{1}^{\dagger} H_{2} \Delta_{p}+\frac{1}{2} H_{1}^{\dagger} H_{1} \Delta_{3}+\frac{1}{\sqrt{2}} H_{2}^{\dagger} H_{1} \Delta_{m}-\frac{1}{2} H_{2}^{\dagger} H_{2} \Delta_{3}\right) \\
& -M_{\Phi \Delta}\left(\frac{1}{\sqrt{2}} \Phi_{1}^{*} \Phi_{2} \Delta_{p}+\frac{1}{2} \Phi_{1}^{*} \Phi_{1} \Delta_{3}+\frac{1}{\sqrt{2}} \Phi_{2}^{*} \Phi_{1} \Delta_{m}-\frac{1}{2} \Phi_{2}^{*} \Phi_{2} \Delta_{3}\right) \\
& +\lambda_{H \Delta}\left(H_{1}^{\dagger} H_{1}+H_{2}^{\dagger} H_{2}\right)\left(\frac{1}{2} \Delta_{3}^{2}+\Delta_{p} \Delta_{m}\right) \\
& +\lambda_{H \Phi}\left(H_{1}^{\dagger} H_{1}+H_{2}^{\dagger} H_{2}\right)\left(\Phi_{1}^{*} \Phi_{1}+\Phi_{2}^{*} \Phi_{2}\right) \\
& +\lambda_{\Phi \Delta}\left(\Phi_{1}^{*} \Phi_{1}+\Phi_{2}^{*} \Phi_{2}\right)\left(\frac{1}{2} \Delta_{3}^{2}+\Delta_{p} \Delta_{m}\right)
\end{aligned}
$$

where

$$
\Delta_{H}=\left(\begin{array}{cc}
\Delta_{3} / 2 & \Delta_{p} / \sqrt{2} \\
\Delta_{m} / \sqrt{2} & -\Delta_{3} / 2
\end{array}\right) \text { with } \Delta_{m}=\left(\Delta_{p}\right)^{*} \text { and }\left(\Delta_{3}\right)^{*}=\Delta_{3},
$$

and $\Phi_{H}=\left(\begin{array}{ll}\Phi_{1} & \Phi_{2}\end{array}\right)^{T}$.

\footnotetext{
${ }^{1}$ Here, we consider renormalizable terms only. In addition, $\mathrm{SU}(2)_{L}$ multiplication is implicit and suppressed.
} 
At this point we would like to make some general comments for the above potential before performing the minimization of it to achieve spontaneous symmetry breaking (see next section).

- $\mathrm{U}(1)_{X}$ is introduced to simplify the Higgs potential $V\left(H, \Delta_{H}, \Phi_{H}\right)$ in eq. (2.1). For example, a term $\Phi_{H}^{T} \Delta_{H} \Phi_{H}$ obeying the $\mathrm{SU}(2)_{H}$ symmetry would be allowed in the absence of $\mathrm{U}(1)_{X}$. Note that as far as the scalar potential is concerned, treating $\mathrm{U}(1)_{X}$ as a global symmetry is sufficient to kill this and other unwanted terms.

- In eq. (2.4), if $-\mu_{\Delta}^{2}<0, \mathrm{SU}(2)_{H}$ is spontaneously broken by the vev $\left\langle\Delta_{3}\right\rangle=-v_{\Delta} \neq 0$ with $\left\langle\Delta_{p, m}\right\rangle=0$ by applying an $\mathrm{SU}(2)_{H}$ rotation.

- The quadratic terms for $H_{1}$ and $H_{2}$ have the following coefficients

$$
\mu_{H}^{2} \mp \frac{1}{2} M_{H \Delta} \cdot v_{\Delta}+\frac{1}{2} \lambda_{H \Delta} \cdot v_{\Delta}^{2}+\frac{1}{2} \lambda_{H \Phi} \cdot v_{\Phi}^{2},
$$

respectively. Thus even with a positive $\mu_{H}^{2}, H_{1}$ can still develop a vev $(0 v / \sqrt{2})^{T}$ breaking $\mathrm{SU}(2)_{L}$ provided that the second term is dominant, while $H_{2}$ remains zero vev. Electroweak symmetry breaking is triggered by the $\mathrm{SU}(2)_{H}$ breaking. Since the doublet $\mathrm{H}_{2}$ does not obtain a vev, its lowest mass component can be potentially a DM candidate whose stability is protected by the gauge group $\mathrm{SU}(2)_{H}$.

- Similarly, the quadratic terms for two fields $\Phi_{1}$ and $\Phi_{2}$ have the coefficients

$$
\mu_{\Phi}^{2} \pm \frac{1}{2} M_{\Phi \Delta} \cdot v_{\Delta}+\frac{1}{2} \lambda_{\Phi \Delta} \cdot v_{\Delta}^{2}+\frac{1}{2} \lambda_{H \Phi} \cdot v^{2},
$$

respectively. The field $\Phi_{2}$ may acquire nontrivial vev and $\left\langle\Phi_{1}\right\rangle=0$ with the help of a large second term.

\subsection{Yukawa couplings}

We start from the quark sector. Setting the quark $\mathrm{SU}(2)_{L}$ doublet, $Q_{L}$, to be an $\mathrm{SU}(2)_{H}$ singlet and including additional $\mathrm{SU}(2)_{L} \operatorname{singlets} u_{R}^{H}$ and $d_{R}^{H}$ which together with the SM righthanded quarks $u_{R}$ and $d_{R}$, respectively, to form $\mathrm{SU}(2)_{H}$ doublets, i.e., $U_{R}^{T}=\left(\begin{array}{ll}u_{R} & u_{R}^{H}\end{array}\right)_{2 / 3}$ and $D_{R}^{T}=\left(\begin{array}{ll}d_{R}^{H} & d_{R}\end{array}\right)_{-1 / 3}$, where the subscript represents hypercharge, we have ${ }^{2}$

$$
\begin{aligned}
\mathcal{L}_{\text {Yuk }} & \supset y_{d} \bar{Q}_{L}\left(D_{R} \cdot H\right)+y_{u} \bar{Q}_{L}\left(U_{R} \cdot \tilde{\tilde{H}}\right)+\text { H.c. } \\
& =y_{d} \bar{Q}_{L}\left(d_{R}^{H} H_{2}-d_{R} H_{1}\right)-y_{u} \bar{Q}_{L}\left(u_{R} \tilde{H}_{1}+u_{R}^{H} \tilde{H}_{2}\right)+\text { H.c. },
\end{aligned}
$$

where $\widetilde{\widetilde{H}} \equiv\left(\tilde{H}_{2}-\tilde{H}_{1}\right)^{T}$ with $\tilde{H}_{1,2}=i \tau_{2} H_{1,2}^{*}$. After the EW symmetry breaking $\left\langle H_{1}\right\rangle \neq 0$, $u$ and $d$ obtain their masses but $u_{R}^{H}$ and $d_{R}^{H}$ remain massless since $H_{2}$ does not get a vev.

To give a mass to the additional species, we employ the $\mathrm{SU}(2)_{H}$ scalar doublet $\Phi_{H}=$ $\left(\Phi_{1} \Phi_{2}\right)^{T}$, which is singlet under $\mathrm{SU}(2)_{L}$, and left-handed $\mathrm{SU}(2)_{L, H}$ singlets $\chi_{u}$ and $\chi_{d}$ as

$$
\begin{aligned}
\mathcal{L}_{\text {Yuk }} & \supset-y_{d}^{\prime} \bar{\chi}_{d}\left(D_{R} \cdot \Phi_{H}\right)+y_{u}^{\prime} \bar{\chi}_{u}\left(U_{R} \cdot \tilde{\Phi}_{H}\right)+\text { H.c. } \\
& =-y_{d}^{\prime} \bar{\chi}_{d}\left(d_{R}^{H} \Phi_{2}-d_{R} \Phi_{1}\right)-y_{u}^{\prime} \bar{\chi}_{u}\left(u_{R} \Phi_{1}^{*}+u_{R}^{H} \Phi_{2}^{*}\right)+\text { H.c. },
\end{aligned}
$$

\footnotetext{
${ }^{2} A \cdot B$ is defined as $\epsilon_{i j} A^{i} B^{j}$ where $A$ and $B$ are two 2-dimensional spinor representations of $\mathrm{SU}(2)_{H}$.
} 
where $\Phi$ has $Y=0, Y\left(\chi_{u}\right)=Y\left(U_{R}\right)=2 / 3$ and $Y\left(\chi_{d}\right)=Y\left(D_{R}\right)=-1 / 3$ with $\tilde{\Phi}_{H}=\left(\Phi_{2}^{*}-\right.$ $\left.\Phi_{1}^{*}\right)^{T}$. With $\left\langle\Phi_{2}\right\rangle=v_{\Phi} / \sqrt{2}, u_{R}^{H}\left(\chi_{u}\right)$ and $d_{R}^{H}\left(\chi_{d}\right)$ obtain masses $y_{u}^{\prime} v_{\Phi} / \sqrt{2}$ and $y_{d}^{\prime} v_{\Phi} / \sqrt{2}$, respectively. Note that both $v_{\Delta}$ and $v_{\Phi}$ contribute the $\mathrm{SU}(2)_{H}$ gauge boson masses.

The lepton sector is similar to the quark sector as

$$
\begin{aligned}
\mathcal{L}_{\text {Yuk }} \supset & y_{e} \bar{L}_{L}\left(E_{R} \cdot H\right)+y_{\nu} \bar{L}_{L}\left(N_{R} \cdot \tilde{\tilde{H}}\right)-y_{e}^{\prime} \bar{\chi}_{e}\left(E_{R} \cdot \Phi_{H}\right)+y_{\nu}^{\prime} \bar{\chi}_{\nu}\left(N_{R} \cdot \tilde{\Phi}_{H}\right)+\text { H.c. } \\
= & y_{e} \bar{L}_{L}\left(e_{R}^{H} H_{2}-e_{R} H_{1}\right)-y_{\nu} \bar{L}_{L}\left(\nu_{R} \tilde{H}_{1}+\nu_{R}^{H} \tilde{H}_{2}\right) \\
& -y_{e}^{\prime} \bar{\chi}_{e}\left(e_{R}^{H} \Phi_{2}-e_{R} \Phi_{1}\right)-y_{\nu}^{\prime} \bar{\chi}_{\nu}\left(\nu_{R} \Phi_{1}^{*}+\nu_{R}^{H} \Phi_{2}^{*}\right)+\text { H.c. }
\end{aligned}
$$

where $E_{R}^{T}=\left(e_{R}^{H} e_{R}\right)_{-1}, N_{R}^{T}=\left(\nu_{R} \nu_{R}^{H}\right)_{0}$ in which $\nu_{R}$ and $\nu_{R}^{H}$ are the right-handed neutrino and its $\mathrm{SU}(2)_{H}$ partner respectively, while $\chi_{e}$ and $\chi_{\nu}$ are $\mathrm{SU}(2)_{L, H} \operatorname{singlets}$ with $Y\left(\chi_{e}\right)=-1$ and $Y\left(\chi_{\nu}\right)=0$ respectively. Notice that neutrinos are purely Dirac in this setup, i.e., $\nu_{R}$ paired up with $\nu_{L}$ having Dirac mass $M_{D}^{\nu}=y_{\nu} v / \sqrt{2}$, while $\nu_{R}^{H}$ paired up with $\chi_{\nu}$ having Dirac mass $M_{D}^{\nu^{H}}=y_{\nu}^{\prime} v_{\Phi} / \sqrt{2}$. As a result, the lepton number is conserved, implying vanishing neutrinoless double beta decay. In order to generate the observed neutrino masses of order sub-eV, the Yukawa couplings for $\nu_{L}$ and $\nu_{R}$ are extremely small $\left(\sim 10^{-11}\right)$ even compared to the electron Yukawa coupling. The smallness can arise from, for example, the small overlap among wavefunctions along a warped extra dimension [47, 48].

Alternatively, it may be desirable for the neutrinos to have a Majorana mass term which can be easily incorporated by introducing a $\mathrm{SU}(2)_{H}$ scalar triplet $\Delta_{N}$ with $X\left(\Delta_{N}\right)=-2$. Then a renormalizable term $g_{N} \overline{N_{R}^{c}} \Delta_{N} N_{R}$ with a large $\left\langle\Delta_{N}\right\rangle \neq 0$ will break lepton number and provide large Majorana masses $M_{N}=g_{N}\left\langle\Delta_{N}\right\rangle$ to $\nu_{R} \mathrm{~s}$ (and also $\nu_{R}^{H} \mathrm{~s}$ ). Sub-eV masses for the $\nu_{L} \mathrm{~S}$ can be realized via the type-I seesaw mechanism which allows one large mass of order $M_{N}$ and one small mass of order $\left(M_{D}^{\nu}\right)^{2} / M_{N}$. For $M_{D}^{\nu} \sim y_{\nu} v$ and $v \sim 246 \mathrm{GeV}$, sub-eV neutrino masses can be achieved provided that $y_{\nu} \sim 1.28 \times 10^{-7} \sqrt{M_{N} / \mathrm{GeV}}$.

We note that only one $\mathrm{SU}(2)_{L}$ doublet $H_{1}$ couples to two SM fermion fields in the above Yukawa couplings. The other doublet $H_{2}$ couples to one SM fermion and one non$\mathrm{SM}$ fermion, while the $\mathrm{SU}(2)_{H}$ doublet $\Phi_{H}$ couples to at least one non-SM fermion. As a consequence, there is no flavour changing decays from the SM Higgs in this model. This is in contrast with the $2 \mathrm{HDM}$ where a discrete $Z_{2}$ symmetry needed to be imposed to forbid flavour changing Higgs decays at tree level. Thus, as long as $H_{2}$ does not develop a vev in the parameter space, it is practically an inert Higgs, protected by a local gauge symmetry instead of a discrete one!

\subsection{Anomaly cancellation}

We here demonstrate the aforementioned setup is anomaly-free with respect to both the SM and additional gauge groups. The anomaly cancellation for the SM gauge groups $\mathrm{SU}(3)_{C} \times$ $\mathrm{SU}(2)_{L} \times \mathrm{U}(1)_{Y}$ is guaranteed since addition heavy particles of the same hypercharge form Dirac pairs. Therefore, contributions of the left-handed currents from $\chi_{u}, \chi_{d}, \chi_{\nu}$ and $\chi_{e}$ cancel those of right-handed ones from $u_{R}^{H}, d_{R}^{H}, \nu_{R}^{H}$ and $e_{R}^{H}$ respectively.

Regarding the new gauge group $\mathrm{SU}(2)_{H}$, the only nontrivial anomaly needed to be checked is $\left[\mathrm{SU}(2)_{H}\right]^{2} \mathrm{U}(1)_{Y}$ from the doublets $U_{R}, D_{R}, N_{R}$ and $E_{R}$ with the following 
result

$$
\begin{aligned}
2 \operatorname{Tr}\left[T^{a}\left\{T^{b}, Y\right\}\right] & =2 \delta^{a b}\left(\sum_{l} Y_{l}-\sum_{r} Y_{r}\right)=-2 \delta^{a b} \sum_{r} Y_{r} \\
& =-2 \delta^{a b}\left(3 \cdot 2 \cdot Y\left(U_{R}\right)+3 \cdot 2 \cdot Y\left(D_{R}\right)+2 \cdot Y\left(N_{R}\right)+2 \cdot Y\left(E_{R}\right)\right)
\end{aligned}
$$

where 3 comes from the $\mathrm{SU}(3)_{C}$ color factor and 2 from 2 components in an $\mathrm{SU}(2)_{H}$ doublet. With the quantum number assignment for the various fields listed in table 1, one can check that this anomaly coefficient vanishes for each generation.

In terms of $\mathrm{U}(1)_{X}$, one has to check $\left[\mathrm{SU}(3)_{C}\right]^{2} \mathrm{U}(1)_{X},\left[\mathrm{SU}(2)_{H}\right]^{2} \mathrm{U}(1)_{X},\left[\mathrm{U}(1)_{X}\right]^{3}$, $\left[\mathrm{U}(1)_{Y}\right]^{2} \mathrm{U}(1)_{X}$ and $\left[\mathrm{U}(1)_{X}\right]^{2} \mathrm{U}(1)_{Y}{ }^{3}$ The first three terms are zero due to cancellation between $U_{R}$ and $D_{R}$ and between $E_{R}$ and $N_{R}$ with opposite $\mathrm{U}(1)_{X}$ charges. For $\left[\mathrm{U}(1)_{Y}\right]^{2} \mathrm{U}(1)_{X}$ and $\left[\mathrm{U}(1)_{X}\right]^{2} \mathrm{U}(1)_{Y}$, one has respectively

$$
\begin{aligned}
& 2 \cdot\left(3 \cdot\left(Y\left(U_{R}\right)^{2} X\left(U_{R}\right)+Y\left(D_{R}\right)^{2} X\left(D_{R}\right)\right)+Y\left(E_{R}\right)^{2} X\left(E_{R}\right)\right), \\
& 2 \cdot\left(3 \cdot\left(X\left(U_{R}\right)^{2} Y\left(U_{R}\right)+X\left(D_{R}\right)^{2} Y\left(D_{R}\right)\right)+X\left(E_{R}\right)^{2} Y\left(E_{R}\right)\right),
\end{aligned}
$$

both of which vanish.

One can also check the perturbative gravitational anomaly [49] associated with the hypercharge and $\mathrm{U}(1)_{X}$ charge current couples to two gravitons is proportional to the following sum of the hypercharge

$$
\begin{aligned}
3 \cdot\left(2 \cdot Y\left(Q_{L}\right)\right. & \left.+Y\left(\chi_{u}\right)+Y\left(\chi_{d}\right)-2 \cdot Y\left(U_{R}\right)-2 \cdot Y\left(D_{R}\right)\right) \\
& +2 \cdot Y\left(L_{L}\right)+Y\left(\chi_{\nu}\right)+Y\left(\chi_{e}\right)-2 \cdot Y\left(N_{R}\right)-2 \cdot Y\left(E_{R}\right)
\end{aligned}
$$

and $\mathrm{U}(1)_{X}$ charge

$$
X\left(U_{R}\right)+X\left(D_{R}\right)+X\left(E_{R}\right)+X\left(N_{R}\right),
$$

which also vanish for each generation.

Since there are 4 chiral doublets for $\mathrm{SU}(2)_{L}$ and also 8 chiral doublets for $\mathrm{SU}(2)_{H}$ for each generation, the model is also free of the global $\mathrm{SU}(2)$ anomaly [50] which requires the total number of chiral doublets for any local SU(2) must be even.

We end this section by pointing out that one can also introduce $Q_{L}^{H}=\left(u_{L}^{H} d_{L}^{H}\right)^{T}$ to pair up with $Q_{L}$ and $L_{L}^{H}=\left(\nu_{L}^{H} e_{L}^{H}\right)^{T}$ to pair up with $L_{L}$ to form $\mathrm{SU}(2)_{H}$ doublets. Such possibility is also interesting and will be discussed elsewhere.

\section{Spontaneous symmetry breaking and mass spectra}

After specifying the model content and fermion mass generation, we now switch to the scalar and gauge boson sector. We begin by studying the minimization conditions for spontaneous symmetry breaking, followed by investigating scalar and gauge boson mass spectra. Special attention is paid to mixing effects on both the scalars and gauge bosons.

\footnotetext{
${ }^{3}\left[\mathrm{SU}(2)_{L}\right]^{2} \mathrm{U}(1)_{X}$ anomaly does not exist since fermions charged under $\mathrm{U}(1)_{X}$ are singlets under $\mathrm{SU}(2)_{L}$.
} 


\subsection{Spontaneous symmetry breaking}

To facilitate spontaneous symmetry breaking, let us shift the fields as follows

$$
H_{1}=\left(\begin{array}{c}
G^{+} \\
\frac{v+h}{\sqrt{2}}+i G^{0}
\end{array}\right), \Phi_{H}=\left(\begin{array}{c}
G_{H}^{p} \\
\frac{v_{\Phi}+\phi_{2}}{\sqrt{2}}+i G_{H}^{0}
\end{array}\right), \Delta_{H}=\left(\begin{array}{cc}
\frac{-v_{\Delta}+\delta_{3}}{2} & \frac{1}{\sqrt{2}} \Delta_{p} \\
\frac{1}{\sqrt{2}} \Delta_{m} & \frac{v_{\Delta}-\delta_{3}}{2}
\end{array}\right)
$$

and $H_{2}=\left(H_{2}^{+} H_{2}^{0}\right)^{T}$. Here $v, v_{\Phi}$ and $v_{\Delta}$ are vevs to be determined by minimization of the potential; $\Psi_{G} \equiv\left\{G^{+}, G^{3}, G_{H}^{p}, G_{H}^{0}\right\}$ are Goldstone bosons, to be absorbed by the longitudinal components of $W^{+}, W^{3}, W^{p}, W^{\prime 3}$ respectively; and $\Psi \equiv\left\{h, H_{2}, \Phi_{1}, \phi_{2}, \delta_{3}, \Delta_{p}\right\}$ are the physical fields.

Substituting the vevs in the potential $V$ in eq. (2.1) leads to

$$
\begin{aligned}
V\left(v, v_{\Delta}, v_{\Phi}\right)= & \frac{1}{4}\left[\lambda_{H} v^{4}+\lambda_{\Phi} v_{\Phi}^{4}+\lambda_{\Delta} v_{\Delta}^{4}+2\left(\mu_{H}^{2} v^{2}+\mu_{\Phi}^{2} v_{\Phi}^{2}-\mu_{\Delta}^{2} v_{\Delta}^{2}\right)\right. \\
& \left.-\left(M_{H \Delta} v^{2}+M_{\Phi \Delta} v_{\Phi}^{2}\right) v_{\Delta}+\lambda_{H \Phi} v^{2} v_{\Phi}^{2}+\lambda_{H \Delta} v^{2} v_{\Delta}^{2}+\lambda_{\Phi \Delta} v_{\Phi}^{2} v_{\Delta}^{2}\right]
\end{aligned}
$$

Minimization of the potential in eq. (3.2) leads to the following three equations for the vevs

$$
\begin{aligned}
v \cdot\left(2 \lambda_{H} v^{2}+2 \mu_{H}^{2}-M_{H \Delta} v_{\Delta}+\lambda_{H \Phi} v_{\Phi}^{2}+\lambda_{H \Delta} v_{\Delta}^{2}\right) & =0, \\
v_{\Phi} \cdot\left(2 \lambda_{\Phi} v_{\Phi}^{2}+2 \mu_{\Phi}^{2}-M_{\Phi \Delta} v_{\Delta}+\lambda_{H \Phi} v^{2}+\lambda_{\Phi \Delta} v_{\Delta}^{2}\right) & =0, \\
4 \lambda_{\Delta} v_{\Delta}^{3}-4 \mu_{\Delta}^{2} v_{\Delta}-M_{H \Delta} v^{2}-M_{\Phi \Delta} v_{\Phi}^{2}+2 v_{\Delta}\left(\lambda_{H \Delta} v^{2}+\lambda_{\Phi \Delta} v_{\Phi}^{2}\right) & =0 .
\end{aligned}
$$

Note that one can solve for the non-trivial solutions for $v^{2}$ and $v_{\Phi}^{2}$ in terms of $v_{\Delta}$ and other parameters using eqs. (3.3) and (3.4). Substitute these solutions of $v^{2}$ and $v_{\Phi}^{2}$ into eq. (3.5) leads to a cubic equation for $v_{\Delta}$ which can be solved analytically (See appendix A).

\subsection{Scalar mass spectrum}

The scalar boson mass spectrum can be obtained from taking the second derivatives of the potential with respect to the various fields and evaluate it at the minimum of the potential. The mass matrix thus obtained contains three diagonal blocks. The first block is $3 \times 3$. In the basis of $S=\left\{h, \delta_{3}, \phi_{2}\right\}$ it is given by

$$
\mathcal{M}_{0}^{2}=\left(\begin{array}{ccc}
2 \lambda_{H} v^{2} & \frac{v}{2}\left(M_{H \Delta}-2 \lambda_{H \Delta} v_{\Delta}\right) & \lambda_{H \Phi} v v_{\Phi} \\
\frac{v}{2}\left(M_{H \Delta}-2 \lambda_{H \Delta} v_{\Delta}\right) & \frac{1}{4 v_{\Delta}}\left(8 \lambda_{\Delta} v_{\Delta}^{3}+M_{H \Delta} v^{2}+M_{\Phi \Delta} v_{\Phi}^{2}\right) & \frac{v_{\Phi}}{2}\left(M_{\Phi \Delta}-2 \lambda_{\Phi \Delta} v_{\Delta}\right) \\
\lambda_{H \Phi} v v_{\Phi} & \frac{v_{\Phi}}{2}\left(M_{\Phi \Delta}-2 \lambda_{\Phi \Delta} v_{\Delta}\right) & 2 \lambda_{\Phi} v_{\Phi}^{2}
\end{array}\right) .
$$

This matrix can be diagonalized by a similar transformation with orthogonal matrix $O$, which defined as $|f\rangle_{i} \equiv O_{i j}|m\rangle_{j}$ with $i$ and $j$ referring to the flavour and mass eigenstates respectively,

$$
O^{T} \cdot \mathcal{M}_{0}^{2} \cdot O=\operatorname{Diag}\left(m_{h_{1}}^{2}, m_{h_{2}}^{2}, m_{h_{3}}^{2}\right),
$$

where the three eigenvalues are in ascending order. The lightest eigenvalue $m_{h_{1}}$ will be identified as the $125 \mathrm{GeV}$ Higgs $h_{1}$ observed at the LHC and the other two $m_{h_{2}}$ and $m_{h_{3}}$ 
are for the heavier Higgses $h_{2}$ and $h_{3}$. The physical Higgs $h_{i}$ is a linear combination of the three components of $S: h_{i}=O_{j i} S_{j}$. Thus the $125 \mathrm{GeV}$ scalar boson could be a mixture of the neutral components of $H_{1}$ and the $\mathrm{SU}(2)_{H}$ doublet $\Phi_{H}$, as well as the real component $\delta_{3}$ of the $\mathrm{SU}(2)_{H}$ triplet $\Delta_{H}$.

The SM Higgs $h_{1}$ tree-level couplings to $f \bar{f}, W^{+} W^{-}, Z Z$ and $H_{2}^{+} H_{2}^{-}$pairs, each will be modified by an overall factor of $O_{11}$, resulting a reduction by $\left|O_{11}\right|^{2}$ on the $h_{1}$ decay branching ratios into these channels. On the other hand, as we shall see later, $h_{1} \rightarrow \gamma \gamma$ and $Z \gamma$ involve extra contributions from the $\delta_{3}$ and $\phi_{2}$ components, which could lead to either enhancement or suppression with respect to the SM prediction.

The second block is also $3 \times 3$. In the basis of $G=\left\{G_{H}^{p}, \Delta_{p}, H_{2}^{0 *}\right\}$ it is given by

$$
\mathcal{M}_{0}^{\prime 2}=\left(\begin{array}{ccc}
M_{\Phi} v_{\Delta} & -\frac{1}{2} M_{\Phi \Delta} v_{\Phi} & 0 \\
-\frac{1}{2} M_{\Phi \Delta} v_{\Phi} & \frac{1}{4 v_{\Delta}}\left(M_{H \Delta} v^{2}+M_{\Phi \Delta} v_{\Phi}^{2}\right) & \frac{1}{2} M_{H \Delta} v \\
0 & \frac{1}{2} M_{H \Delta} v & M_{H \Delta} v_{\Delta}
\end{array}\right)
$$

It is easy to show that eq. (3.8) has a zero eigenvalue, associated with the physical Goldstone boson, which is a mixture of $G_{H}^{p}, \Delta_{p}$ and $H_{2}^{0 *}$. The other two eigenvalues are the masses of two physical fields $\widetilde{\Delta}$ and $D$. They are given by

$$
\begin{aligned}
M_{\widetilde{\Delta}, D}= & \frac{1}{8 v_{\Delta}}\left\{M_{H \Delta} v^{2}+4\left(M_{H \Delta}+M_{\Phi \Delta}\right) v_{\Delta}^{2}+M_{\Phi \Delta} v_{\Phi}^{2}\right. \\
& \left. \pm\left[\left(M_{H \Delta}\left(v^{2}+4 v_{\Delta}^{2}\right)+M_{\Phi \Delta}\left(v_{\Phi}^{2}+4 v_{\Delta}^{2}\right)\right)^{2}-16 M_{H \Delta} M_{\Phi \Delta} v_{\Delta}^{2}\left(v^{2}+4 v_{\Delta}^{2}+v_{\Phi}^{2}\right)\right]^{\frac{1}{2}}\right\} .
\end{aligned}
$$

$D$ can be a DM candidate in G2HDM. Note that in the parameter space where the quantity inside the square root of eq. (3.9) is very small, $\widetilde{\Delta}$ would be degenerate with $D$. In this case, we need to include coannihilation processes for relic density calculation. Moreover, it is possible in our model to have $\nu_{R}^{H}$ or $\chi_{\nu}\left(\nu_{R}\right.$ either is too light or is not stable since it decays to SM lepton and Higgs) to be DM candidate as well.

The final block is $4 \times 4$ diagonal, giving

$$
m_{H_{2}^{ \pm}}^{2}=M_{H \Delta} v_{\Delta}
$$

for the physical charged Higgs $H_{2}^{ \pm}$, and

$$
m_{G^{ \pm}}^{2}=m_{G^{0}}^{2}=m_{G_{H}^{0}}^{2}=0,
$$

for the three Goldstone boson fields $G^{ \pm}, G^{0}$ and $G_{H}^{0}$. Note that we have used the minimization conditions eqs. (3.3), (3.4) and (3.5) to simplify various matrix elements of the above mass matrices.

Altogether we have 6 Goldstone particles in the scalar mass spectrum, we thus expect to have two massless gauge particles left over after spontaneous symmetry breaking. One is naturally identified as the photon while the other one could be interpreted as dark photon $\gamma_{D}$. 


\section{3 $\mathrm{SU}(2)_{H} \times \mathrm{U}(1)_{X}$ gauge boson mass spectrum}

After investigating the spontaneous symmetry breaking conditions, we now study the mass spectrum of additional gauge bosons. The gauge kinetic terms for the $\Delta_{H}, \Phi$ and $H$ are

$$
\mathcal{L} \supset \operatorname{Tr}\left[\left(D_{\mu}^{\prime} \Delta_{H}\right)^{\dagger}\left(D^{\prime \mu} \Delta_{H}\right)\right]+\left(D_{\mu}^{\prime} \Phi\right)^{\dagger}\left(D^{\prime \mu} \Phi\right)+\left(D_{\mu}^{\prime} H\right)^{\dagger}\left(D^{\prime \mu} H\right)
$$

with

$$
\begin{aligned}
D_{\mu}^{\prime} \Delta_{H} & =\partial_{\mu} \Delta_{H}-i g_{H}\left[W_{\mu}^{\prime}, \Delta_{H}\right], \\
D_{\mu}^{\prime} \Phi & =\left(\partial_{\mu}-i \frac{g_{H}}{\sqrt{2}}\left(W_{\mu}^{\prime p} T^{p}+W_{\mu}^{\prime m} T^{m}\right)-i g_{H} W_{\mu}^{\prime 3} T^{3}-i g_{X} X_{\mu}\right) \cdot \Phi,
\end{aligned}
$$

and

$$
D_{\mu}^{\prime} H=\left(D_{\mu} \cdot 1-i \frac{g_{H}}{\sqrt{2}}\left(W_{\mu}^{\prime p} T^{p}+W_{\mu}^{\prime m} T^{m}\right)-i g_{H} W_{\mu}^{\prime 3} T^{3}-i g_{X} X_{\mu}\right) \cdot H
$$

where $D_{\mu}$ is the $\mathrm{SU}(2)_{L}$ covariant derivative, acting individually on $H_{1}$ and $H_{2}, g_{H}\left(g_{X}\right)$ is the $\mathrm{SU}(2)_{H}\left(\mathrm{U}(1)_{X}\right)$ gauge coupling constant, and

$$
W_{\mu}^{\prime}=\sum_{a=1}^{3} W^{\prime a} T^{a}=\frac{1}{\sqrt{2}}\left(W_{\mu}^{\prime p} T^{p}+W_{\mu}^{\prime m} T^{m}\right)+W_{\mu}^{\prime 3} T^{3},
$$

in which $T^{a}=\tau^{a} / 2\left(\tau^{a}\right.$ are the Pauli matrices acting on the $\mathrm{SU}(2)_{H}$ space $), W_{\mu}^{\prime(p, m)}=$ $\left(W_{\mu}^{\prime 1} \mp i W_{\mu}^{\prime 2}\right) / \sqrt{2}$, and

$$
T^{p}=\frac{1}{2}\left(\tau^{1}+i \tau^{2}\right)=\left(\begin{array}{ll}
0 & 1 \\
0 & 0
\end{array}\right), T^{m}=\frac{1}{2}\left(\tau^{1}-i \tau^{2}\right)=\left(\begin{array}{ll}
0 & 0 \\
1 & 0
\end{array}\right) .
$$

The SM charged gauge boson $W^{ \pm}$obtained its mass entirely from $v$, so it is given by

$$
M_{W^{ \pm}}=\frac{1}{2} g v,
$$

same as the SM.

The $\mathrm{SU}(2)_{H}$ gauge bosons $W^{\prime a}$ and the $\mathrm{U}(1)_{X}$ gauge boson $X$ receive masses from $\left\langle\Delta_{3}\right\rangle,\left\langle H_{1}\right\rangle$ and $\left\langle\Phi_{2}\right\rangle$. The terms contributed from the doublets are similar with that from the standard model. Since $\Delta_{H}$ transforms as a triplet under $\mathrm{SU}(2)_{H}$, i.e., in the adjoint representation, the contribution to the $W^{\prime a}$ masses arise from the term

$$
\mathcal{L} \supset g_{H}^{2} \operatorname{Tr}\left(\left[W^{\prime \mu}, \Delta_{H}\right]^{\dagger}\left[W_{\mu}^{\prime}, \Delta_{H}\right]\right) .
$$

All in all, the $W^{\prime(p, m)}$ receives a mass from $\left\langle\Delta_{3}\right\rangle,\left\langle\Phi_{2}\right\rangle$ and $\left\langle H_{1}\right\rangle$

$$
m_{W^{\prime(p, m)}}^{2}=\frac{1}{4} g_{H}^{2}\left(v^{2}+v_{\Phi}^{2}+4 v_{\Delta}^{2}\right),
$$


while gauge bosons $X$ and $W^{\prime 3}$, together with the $\mathrm{SM} W^{3}$ and $\mathrm{U}(1)_{Y}$ gauge boson $B$, acquire their masses from $\left\langle\Phi_{2}\right\rangle$ and $\left\langle H_{1}\right\rangle$ only but not from $\left\langle\Delta_{H}\right\rangle$ :

$$
\frac{1}{8}\left(v^{2}\left(2 g_{X} X_{\mu}+g_{H} W_{\mu}^{\prime 3}-g W_{\mu}^{3}+g^{\prime} B_{\mu}\right)^{2}+v_{\Phi}^{2}\left(-2 g_{X} X_{\mu}+g_{H} W_{\mu}^{\prime 3}\right)^{2}\right),
$$

where $g^{\prime}$ is the $\mathrm{SM} \mathrm{U}(1)_{Y}$ gauge coupling.

Note that the gauge boson $W^{\prime(p, m)}$ corresponding to the $\mathrm{SU}(2)_{H}$ generators $T^{ \pm}$do not carry the SM electric charge and therefore will not mix with the SM $W^{ \pm}$bosons while $W^{\prime 3}$ and $X$ do mix with the SM $W^{3}$ and $B$ bosons via $\left\langle H_{1}\right\rangle$. In fact, only two of $W^{3}, W^{\prime 3}$, $B$ and $X$ will become massive, by absorbing the imaginary part of $H_{1}^{0}$ and $\Phi_{2}$. To avoid undesired additional massless gauge bosons, one can introduce extra scalar fields charged under only $\mathrm{SU}(2)_{H} \times \mathrm{U}(1)_{X}$ but not under the $\mathrm{SM}$ gauge group to give a mass to $W^{\prime 3}$ and $X$, without perturbing the SM gauge boson mass spectrum. Another possibility is to involve the Stueckelberg mechanism to give a mass to the $\mathrm{U}(1)_{X}$ gauge boson as done in refs. [51-54]. Alternatively, one can set $g_{X}=0$ to decouple $X$ from the theory or simply treat $\mathrm{U}(1)_{X}$ as a global symmetry, after all as mentioned before $\mathrm{U}(1)_{X}$ is introduced to simplify the Higgs potential by forbidding terms like $\Phi_{H}^{T} \Delta_{H} \Phi_{H}$, which is allowed under $\mathrm{SU}(2)_{H}$ but not $\mathrm{U}(1)_{X}$.

From eq. (3.21), one can obtain the following mass matrix for the neutral gauge bosons in the basis $V^{\prime}=\left\{B, W^{3}, W^{\prime 3}, X\right\}$ :

$$
\mathcal{M}_{1}^{2}=\left(\begin{array}{cccc}
\frac{g^{\prime 2} v^{2}}{4} & -\frac{g^{\prime} g v^{2}}{4} & \frac{g^{\prime} g_{H} v^{2}}{4} & \frac{g^{\prime} g_{X} v^{2}}{2} \\
-\frac{g^{\prime} g v^{2}}{4} & \frac{g^{2} v^{2}}{4} & -\frac{g g_{H} v^{2}}{4} & -\frac{g g_{X} v^{2}}{2} \\
\frac{g^{\prime} g_{H} v^{2}}{4} & -\frac{g g_{H} v^{2}}{4} & \frac{g_{H}^{2}\left(v^{2}+v_{\Phi}^{2}\right)}{4} & \frac{g_{H} g_{X}\left(v^{2}-v_{\Phi}^{2}\right)}{2} \\
\frac{g^{\prime} g_{X} v^{2}}{2} & -\frac{g g_{X} v^{2}}{2} & \frac{g_{H} g_{X}\left(v^{2}-v_{\Phi}^{2}\right)}{2} & g_{X}^{2}\left(v^{2}+v_{\Phi}^{2}\right)
\end{array}\right) .
$$

As anticipated, this mass matrix has two zero eigenvalues corresponding to $m_{\gamma}=0$ and $m_{\gamma_{D}}=0$ for the photon and dark photon respectively. The other two nonvanishing eigenvalues are

$$
M_{ \pm}^{2}=\frac{1}{8}\left[\left(\alpha v^{2}+\beta v_{\Phi}^{2}\right) \pm \sqrt{\left(\alpha v^{2}+\beta v_{\Phi}^{2}\right)^{2}-4 v^{2} v_{\Phi}^{2}\left(\alpha \beta-\gamma^{2}\right)}\right]
$$

where

$$
\begin{aligned}
\alpha & =g^{2}+g^{2}+g_{H}^{2}+4 g_{X}^{2} \\
\beta & =g_{H}^{2}+4 g_{X}^{2} \\
\gamma & =g_{H}^{2}-4 g_{X}^{2} .
\end{aligned}
$$

A strictly massless dark photon might not be phenomenologically desirable. One could have a Stueckelberg extension of the above model by including the Stueckelberg mass term $[51,52]$

$$
\mathcal{L}_{\mathrm{Stu}}=+\frac{1}{2}\left(\partial_{\mu} a+M_{X} X_{\mu}+M_{Y} B_{\mu}\right)^{2}
$$


where $M_{X}$ and $M_{Y}$ are the Stueckelberg masses for the gauge fields $X_{\mu}$ and $B_{\mu}$ of $\mathrm{U}(1)_{X}$ and $\mathrm{U}(1)_{Y}$ respectively, and $a$ is the axion field. Thus the neutral gauge boson mass matrix is modified as

$$
\mathcal{M}_{1}^{2}=\left(\begin{array}{cccc}
\frac{g^{\prime 2} v^{2}}{4}+M_{Y}^{2} & -\frac{g^{\prime} g v^{2}}{4} & \frac{g^{\prime} g_{H} v^{2}}{4} & \frac{g^{\prime} g_{X} v^{2}}{2}+M_{X} M_{Y} \\
-\frac{g^{\prime} g v^{2}}{4} & \frac{g^{2} v^{2}}{4} & -\frac{g g_{H} v^{2}}{4} & -\frac{g g_{X} v^{2}}{2} \\
\frac{g^{\prime} g_{H} v^{2}}{4} & -\frac{g g_{H} v^{2}}{4} & \frac{g_{H}^{2}\left(v^{2}+v_{\Phi}^{2}\right)}{4} & \frac{g_{H} g_{X}\left(v^{2}-v_{\Phi}^{2}\right)}{2} \\
\frac{g^{\prime} g_{X} v^{2}}{2}+M_{X} M_{Y} & -\frac{g g_{X} v^{2}}{2} & \frac{g_{H} g_{X}\left(v^{2}-v_{\Phi}^{2}\right)}{2} & g_{X}^{2}\left(v^{2}+v_{\Phi}^{2}\right)+M_{X}^{2}
\end{array}\right) .
$$

It is easy to show that this mass matrix has only one zero mode corresponding to the photon, and three massive modes $Z, Z^{\prime}, Z^{\prime \prime}$. This mass matrix can be diagonalized by an orthogonal matrix. The cubic equation for the three eigenvalues can be written down analytically similar to solving the cubic equation for the vev $v_{\Delta}$ given in the appendix A. However their expressions are not illuminating and will not presented here.

As shown in ref. [52], $M_{Y}$ will induce the mixing between $\mathrm{U}(1)_{Y}$ and $\mathrm{U}(1)_{X}$ and the resulting massless eigenstate, the photon, will contain a $\mathrm{U}(1)_{X}$ component, rendering the neutron charge, $Q_{n}=Q_{u}+2 Q_{d}$, nonzero unless $u$ 's and $d$ 's $\mathrm{U}(1)_{X}$ charges are zero or proportional to their electric charges. In this model, however, none of the two solutions can be satisfied. Besides, left-handed SM fields are singlets under $\mathrm{U}(1)_{X}$ while right-handed ones are charged. It implies the left-handed and right-handed species may have different electric charges if the $\mathrm{U}(1)_{X}$ charge plays a role on the electric charge definition. Here we will set $M_{Y}$ to be zero to maintain the relations $Q=I_{3}+Y$ and $1 / e^{2}=1 / g^{\prime 2}+1 / g^{2}$ same as the SM in order to avoid undesired features. As a result, after making a rotation in the $1-2$ plane by the Weinberg angle $\theta_{w}$, the mass matrix $\mathcal{M}_{1}^{2}$ can transform into a block diagonal matrix with the vanishing first column and first row. The nonzero 3-by-3 block matrix can be further diagonalized by an orthogonal matrix $\mathcal{O}$, characterized by three rotation angles $\left(\theta_{12}, \theta_{23}, \theta_{13}\right)$,

$$
\left(\begin{array}{c}
Z_{\mathrm{SM}} \\
W_{3}^{\prime} \\
X
\end{array}\right)=\mathcal{O}\left(\theta_{12}, \theta_{23}, \theta_{13}\right) \cdot\left(\begin{array}{c}
Z \\
Z^{\prime} \\
Z^{\prime \prime}
\end{array}\right) \text { or }\left(\begin{array}{c}
Z \\
Z^{\prime} \\
Z^{\prime \prime}
\end{array}\right)=\mathcal{O}^{T}\left(\theta_{12}, \theta_{23}, \theta_{13}\right) \cdot\left(\begin{array}{c}
Z_{\mathrm{SM}} \\
W_{3}^{\prime} \\
X
\end{array}\right)
$$

where $Z_{\mathrm{SM}}$ is the SM $Z$ boson without the presence of the $W_{3}^{\prime}$ and $X$ bosons. In this model, the charged current mediated by the $W$ boson and the electric current by the photon $\gamma$ are exactly the same as in the SM:

$$
\begin{aligned}
\mathcal{L}(\gamma) & =\sum_{f} Q_{f} e \bar{f} \gamma^{\mu} f A_{\mu}, \\
\mathcal{L}(W) & =\frac{g}{\sqrt{2}}\left(\overline{\nu_{L}} \gamma^{\mu} e_{L}+\overline{u_{L}} \gamma^{\mu} d_{L}\right) W_{\mu}^{+}+\text {H.c. },
\end{aligned}
$$

where $Q_{f}$ is the corresponding fermion electric charge in units of $e$. On the other hand, neutral current interactions, including ones induced by $W^{\prime}$, take the following form (for illustration, only the lepton sector is shown but it is straightforward to include the quark sector)

$$
\mathcal{L}_{N C}=\mathcal{L}(Z)+\mathcal{L}\left(Z^{\prime}\right)+\mathcal{L}\left(Z^{\prime \prime}\right)+\mathcal{L}\left(W^{\prime}\right)
$$


where

$$
\begin{aligned}
\mathcal{L}(Z) & =g \mathcal{O}_{11} J_{Z_{\mathrm{SM}}}^{\mu}+g_{H} \mathcal{O}_{21} J_{W^{\prime}}^{\mu} Z_{\mu}^{\prime}+g_{X} \mathcal{O}_{31} J_{X}^{\mu} Z_{\mu}^{\prime \prime} \\
\mathcal{L}\left(Z^{\prime}\right) & =g \mathcal{O}_{12} J_{Z_{\mathrm{SM}}}^{\mu}+g_{H} \mathcal{O}_{22} J_{W^{\prime 3}}^{\mu} Z_{\mu}^{\prime}+g_{X} \mathcal{O}_{32} J_{X}^{\mu} Z_{\mu}^{\prime \prime} \\
\mathcal{L}\left(Z^{\prime \prime}\right) & =g \mathcal{O}_{13} J_{Z_{\mathrm{SM}}}^{\mu}+g_{H} \mathcal{O}_{23} J_{W^{\prime}}^{\mu} Z_{\mu}^{\prime}+g_{X} \mathcal{O}_{33} J_{X}^{\mu} Z_{\mu}^{\prime \prime} \\
\mathcal{L}\left(W^{\prime}\right) & =\frac{g_{H}}{\sqrt{2}}\left(\overline{e_{R}^{H}} \gamma^{\mu} e_{R}+\bar{\nu}_{R} \gamma^{\mu} \nu_{R}^{H}\right) W_{\mu}^{\prime p}+\text { H.c. }
\end{aligned}
$$

and

$$
\begin{aligned}
J_{Z_{\mathrm{SM}}}^{\mu}= & \frac{1}{\cos \theta_{w}}\left(\left(\sum_{f=e, \nu} \overline{f_{L}} \gamma^{\mu}\left(I_{3}-Q_{f} \sin \theta_{w}\right) f_{L}+\overline{f_{R}} \gamma^{\mu}\left(-Q_{f} \sin \theta_{w}\right) f_{R}\right)\right. \\
J_{W^{\prime 3}}^{\mu} & \left.=\sum_{f=N_{R}, E_{R}} \overline{e_{R}^{H}} \gamma^{\mu}\left(\sin \theta_{w}\right) e_{R}^{H}\right), \\
J_{X}^{\mu} & =\sum_{f=N_{R}, E_{R}} Q_{X}^{f} \overline{f_{R}} \gamma^{\mu} f_{R},
\end{aligned}
$$

with $I_{3}\left(I_{3}^{H}\right)$ being the $\mathrm{SU}(2)_{L}\left(\mathrm{SU}(2)_{H}\right)$ isospin and $Q_{X}^{f}$ the $\mathrm{U}(1)_{X}$ charge. Detailed analysis of the implications of these extra gauge bosons is important and will be presented elsewhere.

\section{Phenomenology}

In this section, we discuss some phenomenology implications of the model by examining the mass spectra of scalars and gauge bosons, $Z^{\prime}$ constraints from various experiments, and Higgs properties of this model against the LHC measurements on the partial decay widths of the SM Higgs boson, which is $h_{1}$ in the model.

\subsection{Numerical solutions for scalar and gauge boson masses}

We first study the SM Higgs mass $\left(m_{h_{1}}\right)$ dependence on the parameters in the mass matrix in eq. (3.6). As we shall see later the vev $v_{\Phi}$ has to be bigger than $10 \mathrm{TeV}(\gg v=246 \mathrm{GeV})$. In light of LEP measurements on the $e^{+} e^{-} \rightarrow e^{+} e^{-}$cross-section [55], the mass matrix will exhibit block-diagonal structure with the bottom-right 2-by-2 block much bigger than the rest and $h$ basically decouple from $\phi_{2}$ and $\delta_{3}$.

To demonstrate this behavior, we simplify the model by setting $\lambda_{H \Delta}, \lambda_{H \Phi}, \lambda_{\Phi \Delta}$ equal to zero and then choose $\lambda_{\Phi}=0.5, \lambda_{\Delta}=1, v_{\Phi}=v_{\Delta}=10 \mathrm{TeV}$, and $M_{\Phi \Delta}=0.8 M_{H \Delta}$ so that one can investigate how the Higgs mass varies as a function of $\lambda_{H}$ and $M_{H \Delta}$. As shown in figure 1 , when $M_{H \Delta}$ is very small compared to $v_{\Phi}$, the Higgs mass is simply the $(1,1)$ element of the mass matrix, $2 \lambda_{H} v^{2}$, and $h_{1}$ is just $h$, i.e., $O_{11}^{2} \simeq 1$. Nonetheless, when $M_{H \Delta}$ becomes comparable to $v_{\Phi}$, and the $(1,2)$ element of the mass matrix gives rise to a sizable but negative contribution to the Higgs mass, requiring a larger value of $\lambda_{H}$ than 


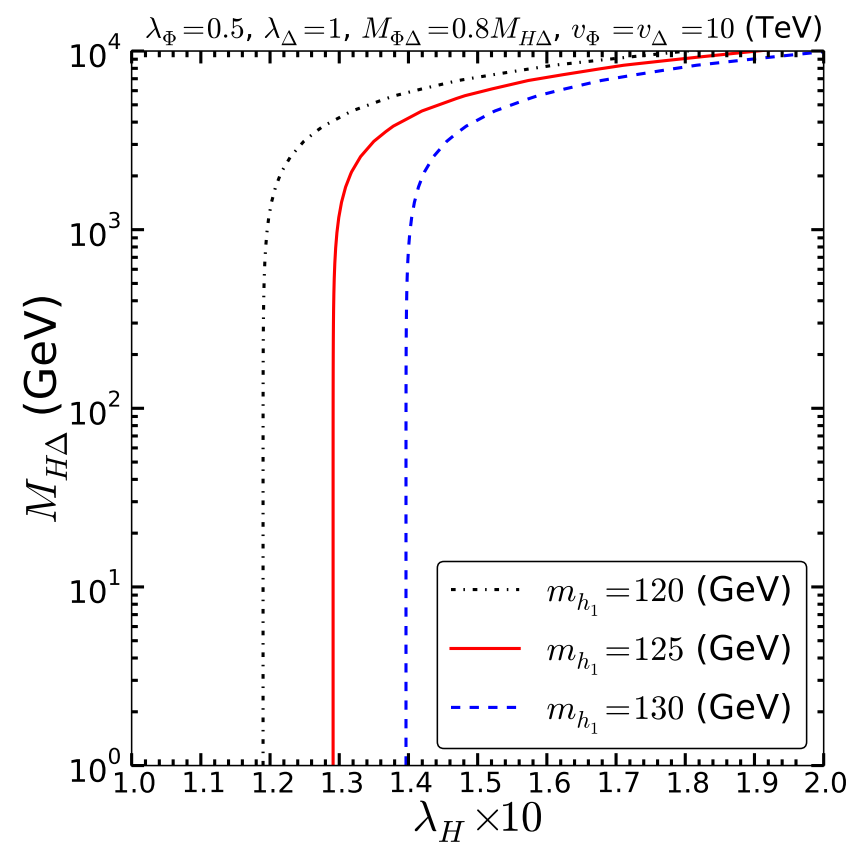

Figure 1. The SM Higgs mass dependence on $M_{H \Delta}$ and $\lambda_{H}$. In the limit of $v_{\Phi}, v_{\Delta} \gg v$, the Higgs mass is basically determined by two parameters $M_{H \Delta}$ and $\lambda_{H}$ only. Other parameters are set as follows: $\lambda_{\Phi}=0.5, \lambda_{\Delta}=1, v_{\Phi}=v_{\Delta}=10 \mathrm{TeV}$ and $M_{\Phi \Delta}=0.8 M_{H \Delta}$.

the $\mathrm{SM}$ one so as to have a correct Higgs mass. In this regime, $\left|O_{11}\right|$ is, however, still very SM-like: $O_{11}^{2} \simeq 1$. Therefore, one has to measure the quartic coupling $\lambda_{H}$ through the double Higgs production to be able to differentiate this model from the SM.

For the analysis above, we neglect the fact all vevs, $v_{\Phi}, v_{\Delta}$ and $v$ are actually functions of the parameters $\mu \mathrm{s}, M \mathrm{~s}$ and $\lambda \mathrm{s}$ in eq. (2.1), the total scalar potential. The analytical solutions of the vevs are collected in appendix A. As a consequence, we now numerically diagonalized the matrices (3.6) and (3.8) as functions of $\mu, M$ and $\lambda$, i.e., replacing all vevs by the input parameters. It is worthwhile to mention that $v_{\Delta}$ has three solutions as eq. (3.5) is a cubic equation for $v_{\Delta}$. Only one of the solutions corresponds to the global minimum of the full scalar potential. We, however, include both the global and local minimum neglecting the stability issue for the latter since it will demand detailed study on the nontrivial potential shape which is beyond the scope of this work.

In order to explore the possibility of a non-SM like Higgs having a $125 \mathrm{GeV}$ mass, we further allow for nonzero mixing couplings. We perform a grid scan with 35 steps of each dimension in the range

$$
\begin{aligned}
10^{-2} & \leq \lambda_{H \Delta} \leq 5 \\
10^{-2} & \leq \lambda_{H \Phi} \leq 5 \\
10^{-2} & \leq \lambda_{\Phi \Delta} \leq 5 \\
10^{-1} & \leq \lambda_{H} \leq 5 \\
1.0 & \leq M_{H \Delta} / \mathrm{GeV} \leq 2 \times 10^{4} \\
1.05 & \leq v_{\Delta} / v_{\Phi} \leq 5.0
\end{aligned}
$$




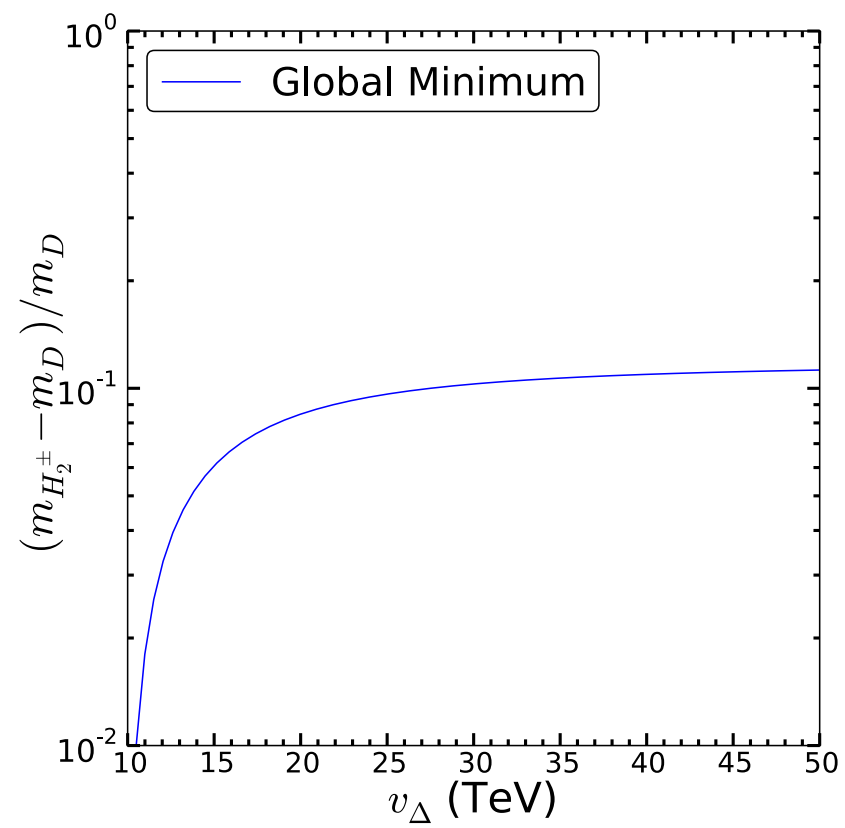

Figure 2. Mass difference of the charged Higgs and dark matter normalized to $m_{D}$ as a function of $v_{\Delta}$ for $M_{\Phi \Delta}=0.8 M_{H \Delta}$ and $v_{\Phi}=10 \mathrm{TeV}$ used in table 2 .

\begin{tabular}{|c|c|c|c|c|c|c|}
\hline & \multicolumn{3}{|c|}{ Global } & \multicolumn{3}{c|}{ Local } \\
\hline Benchmark points & A & B & C & D & E & F \\
\hline$\lambda_{H \Delta}$ & 0.186 & 1.390 & 0.186 & 2.406 & 0.557 & 2.406 \\
\hline$\lambda_{H \Phi}$ & 0.669 & 1.390 & $7.467 \times 10^{-2}$ & 2.004 & $5.181 \times 10^{-2}$ & 0.557 \\
\hline$\lambda_{\Phi \Delta}$ & 1.390 & 1.390 & $3.594 \times 10^{-2}$ & $2.077 \times 10^{-2}$ & 1.390 & 0.386 \\
\hline$\frac{v_{\Delta}}{\mathrm{TeV}}$ & 10.993 & 10.993 & 12.050 & 10.5 & 15.870 & 10.993 \\
\hline$\lambda_{H}$ & 4.456 & 3.155 & 0.141 & 3.540 & 1.775 & 1.582 \\
\hline$\frac{M_{H} \Delta}{\mathrm{GeV}}$ & 24.632 & 59.021 & 1.0 & 1.0 & 78.979 & 18.408 \\
\hline$\frac{m_{h 1}}{\mathrm{GeV}}$ & 125.133 & 124.988 & 125.384 & 125.049 & 124.953 & 125.073 \\
$\frac{m_{h 2}}{\mathrm{TeV}}$ & 1.717 & 1.692 & 9.995 & 10.009 & 1.783 & 9.394 \\
$\frac{m_{h 3}}{\mathrm{TeV}}$ & 1.842 & 18.420 & 17.044 & 14.857 & 24.513 & 15.926 \\
$\frac{m_{D}}{\mathrm{GeV}}$ & 511.162 & 791.244 & 106.287 & 101.447 & 1049.883 & 441.884 \\
$\frac{m_{H}}{\mathrm{GeV}}$ & 520.375 & 805.504 & 109.773 & 102.469 & 1119.586 & 449.848 \\
$\mathcal{O}_{11}^{2}$ & 0.823 & 0.897 & 0.999 & 0.996 & 0.939 & 0.999 \\
$R_{\gamma \gamma}$ & 0.820 & 0.895 & 0.920 & 0.903 & 0.938 & 0.995 \\
$R_{\gamma Z}$ & 0.822 & 0.896 & 0.968 & 0.958 & 0.938 & 0.997 \\
\hline
\end{tabular}

Table 2. Six representative benchmark points. We fix other parameters as $\lambda_{\Phi}=0.5, \lambda_{\Delta}=1$, $v_{\Phi}=10 \mathrm{TeV}$, and $M_{\Phi \Delta}=0.8 M_{H \Delta}$.

In order not to overcomplicate the analysis, from now on we make $\lambda_{\Phi}=0.5, \lambda_{\Delta}=1$, $v_{\Phi}=10 \mathrm{TeV}$, and $M_{\Phi \Delta}=0.8 M_{H \Delta}$, unless otherwise stated. In table 2 , we show 6 representative benchmark points (3 global and 3 local minima) from our grid scan with the dark matter mass $m_{D}$ and the charged Higgs $m_{H^{ \pm}}$of order few hundred GeVs, testable 


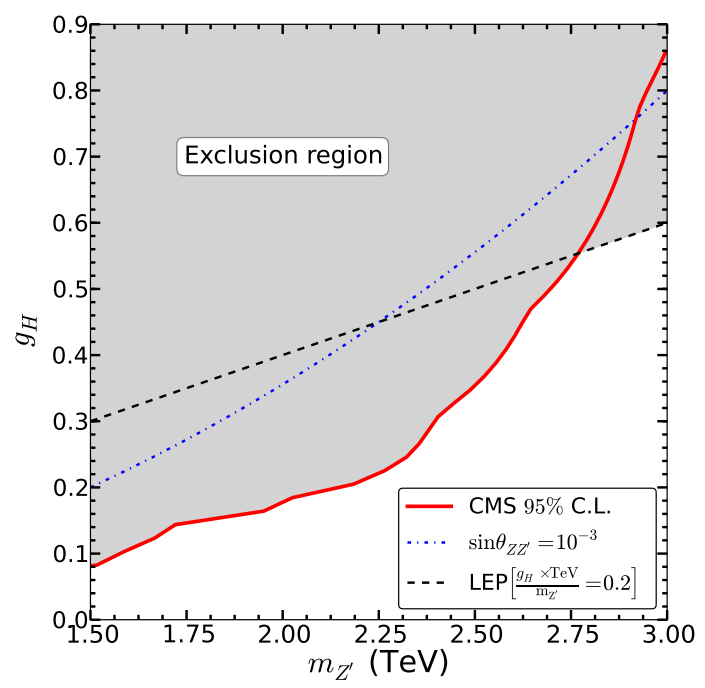

Figure 3. Constraints from the direct $Z^{\prime}$ resonance search based on dilepton channels (red line) at the LHC, from $Z-Z^{\prime}$ mixing (blue dotted line) and the LEP constraints on the electron-positron scattering cross-section (black dashed line) in the $m_{Z^{\prime}}-g_{H}$ plane.

in the near future. It is clear that Global scenario can have the SM Higgs composition significantly different from 1 , as $\mathcal{O}_{11}^{2} \sim 0.8$ in benchmark point $\mathrm{A}$, but $h_{1}$ is often just $h$ in Local case. On the other hand, the other two heavy Higgses are as heavy as TeV because their mass are basically determined by $v_{\Delta}$ and $v_{\Phi}$.

For the other mass matrix eq. (3.8), we focus on the mass splitting between the dark matter and the charged Higgs and it turns out the mass splitting mostly depends on $v_{\Phi}$ and $v_{\Delta}$. In figure 2, we present the mass difference normalized to $m_{D}$ as a function of $v_{\Delta}$ for $M_{\Phi \Delta}=0.8 M_{H \Delta}$ and $v_{\Phi}=10 \mathrm{TeV}$ used in table 2 . The behavior can be easily understood as

$$
\begin{aligned}
\frac{m_{H_{2}^{ \pm}}-m_{D}}{m_{D}} & \simeq \frac{v_{\Delta}^{2}-v_{\Phi}^{2}+\left|v_{\Delta}^{2}-v_{\Phi}^{2}\right|}{8 v_{\Delta}^{2}+2 v_{\Phi}^{2}} \\
& =\frac{1-v_{\Phi}^{2} / v_{\Delta}^{2}}{4+v_{\Phi}^{2} / v_{\Delta}^{2}}
\end{aligned}
$$

where we have neglected terms involving $v$ since we are interested in the limit of $v_{\Delta}, v_{\Phi} \gg v$. Note that this result is true as long as $M_{\Phi \Delta}=0.8 M_{H \Delta}$ and $v_{\Delta}>v_{\Phi} \gg v$ regardless of the other parameters.

\section{$4.2 \quad Z^{\prime}$ constraints}

Since performing a full analysis of the constraints on extra neutral gauge bosons including all possible mixing effects from $\gamma, Z, Z^{\prime}, Z^{\prime \prime}$ is necessarily complicated, we will be contented in this work by a simple scenario discussed below.

The neutral gauge boson mass matrix in eq. (3.22) can be simplified a lot by making $\mathrm{U}(1)_{X}$ a global symmetry. In the limit of $g_{X}=M_{X}=0$, the $\mathrm{U}(1)_{X}$ gauge boson $X$ 
decouples from the theory, and the 3-by-3 mass matrix in the basis of $B, W^{3}$ and $W^{\prime 3}$ can be rotated into the mass basis of the $\mathrm{SM} \gamma, Z$ and the $\mathrm{SU}(2)_{H} Z^{\prime}$ by:

$$
\begin{aligned}
\left(\begin{array}{ccc}
m_{\gamma}^{2} & 0 & 0 \\
0 & m_{Z}^{2} & 0 \\
0 & 0 & m_{Z^{\prime}}^{2}
\end{array}\right)=R_{23}\left(\theta_{\left.Z Z^{\prime}\right)^{T} R_{12}\left(\theta_{w}\right)^{T} \times}\right. \\
\times\left(\begin{array}{ccc}
\frac{g^{\prime 2} v^{2}}{4} & -\frac{g^{\prime} g v^{2}}{4} & \frac{g^{\prime} g_{H} v^{2}}{4} \\
-\frac{g^{\prime} g v^{2}}{4} & \frac{g^{2} v^{2}}{4} & -\frac{g g_{H} v^{2}}{4} \\
\frac{g^{\prime} g_{H} v^{2}}{4} & -\frac{g g_{H} v^{2}}{4} & \frac{g_{H}^{2}\left(v^{2}+v_{\Phi}^{2}\right)}{4}
\end{array}\right) R_{12}\left(\theta_{w}\right) R_{23}\left(\theta_{Z Z^{\prime}}\right),
\end{aligned}
$$

where $R_{i j}$ refers to a rotation matrix in the $i-j$ block; e.g., $R_{12}\left(\theta_{w}\right)$ is a rotation along the $z$-axis $\left(W^{\prime 3}\right)$ direction with $\cos \theta_{w}$ as the $(1,1)$ and $(2,2)$ element and $-\sin \theta_{w}$ as the $(1,2)$ element $\left(\sin \theta_{w}\right.$ for $\left.(2,1)\right)$. The mixing angles can be easily obtained as

$$
\begin{aligned}
\sin \theta_{w} & =\frac{g^{\prime}}{\sqrt{g^{2}+g^{\prime 2}}}, \\
\sin \theta_{Z Z^{\prime}} & =\frac{\sqrt{2} \sqrt{g^{2}+g^{\prime 2}} g_{H} v^{2}}{\kappa^{1 / 4}\left(-\left(g^{2}+g^{\prime 2}\right) v^{2}+g_{H}^{2}\left(v^{2}+v_{\Phi}^{2}\right)+\kappa^{1 / 2}\right)^{1 / 2}},
\end{aligned}
$$

where $\kappa=\left(\left(g^{2}+g^{\prime 2}+g_{H}^{2}\right) v^{2}+g_{H}^{2} v_{\Phi}^{2}\right)^{2}-4 g_{H}^{2}\left(g^{2}+g^{\prime 2}\right) v^{2} v_{\Phi}^{2}$. In the limit of $v_{\Phi} \gg v$, we have the approximate result

$$
\sin \theta_{Z Z^{\prime}} \approx \frac{\sqrt{g^{2}+g^{\prime 2}} v^{2}}{g_{H} v_{\Phi}^{2}}
$$

and

$$
m_{Z} \approx \sqrt{g^{2}+g^{\prime 2}} \frac{v}{2}, m_{Z^{\prime}} \approx g_{H} \frac{v_{\Phi}}{2} .
$$

A couple of comments are in order here. First, the SM Weinberg angle characterized by $\theta_{w}$ is unchanged in the presence of $\mathrm{SU}(2)_{H}$. Second, the vev ratio $v^{2} / v_{\Phi}^{2}$ controls the mixing between the $\mathrm{SM} Z$ and $\mathrm{SU}(2)_{H} Z^{\prime}$. However, the $Z-Z^{\prime}$ mixing for $\mathrm{TeV} Z^{\prime}$ is constrained to be roughly less than $0.1 \%$, results from $Z$ resonance line shape measurements [56], electroweak precision test (EWPT) data [57] and collider searches via the $W^{+} W^{-}$final states [58, 59], depending on underlying models.

Direct $Z^{\prime}$ searches based on dilepton channels at the LHC [60-62] yield stringent constraints on the mass of $Z^{\prime}$ of this model since right-handed SM fermions which are part of $\mathrm{SU}(2)_{H}$ doublets couple to the $Z^{\prime}$ boson and thus $Z^{\prime}$ can be produced and decayed into dilepton at the LHC. To implement LHC $Z^{\prime}$ bounds, we take the $Z^{\prime}$ constraint [62] on the Sequential Standard Model (SSM) with SM-like couplings [63], rescaling by a factor of $g_{H}^{2}\left(\cos ^{2} \theta_{w} / g^{2}\right)$. It is because first $\mathrm{SU}(2)_{H}$ does not have the Weinberg angle in the limit of $g_{X}=0$ and second we assume $Z^{\prime}$ decays into the SM fermions only and branching ratios into the heavy fermions are kinematically suppressed, i.e., $Z^{\prime}$ decay branching ratios are similar to those of the SM $Z$ boson. The direct search bound becomes weaker once 
heavy fermion final states are open. Note also that $Z^{\prime}$ couples only to the right-handed SM fields unlike $Z^{\prime}$ in the SSM couples to both left-handed and right-handed fields as in the SM. The SSM $Z^{\prime}$ left-handed couplings are, however, dominant since the right-handed ones are suppressed by the Weinberg angle. Hence we simply rescale the SSM result by $g_{H}^{2}\left(\cos ^{2} \theta_{w} / g^{2}\right)$ without taking into account the minor difference on the chiral structure of the couplings.

In addition, $Z^{\prime}$ also interacts with the right-handed electron and will contribute to $e^{+} e^{-} \rightarrow \ell^{+} \ell^{-}$processes. LEP measurements on the cross-section of $e^{+} e^{-} \rightarrow \ell^{+} \ell^{-}$can be translated into the constraints on the new physics scale in the context of the effective four-fermion interactions [55]

$$
\mathcal{L}_{\text {eff }}=\frac{4 \pi}{(1+\delta) \Lambda^{2}} \sum_{i, j=L, R} \eta_{i, j} \bar{e}_{i} \gamma_{\mu} e_{i} \bar{f}_{j} \gamma^{\mu} f_{j}
$$

where $\delta=0(1)$ for $f \neq e(f=e)$ and $\eta_{i j}=1(-1)$ corresponds to constructive (destructive) interference between the SM and the new physics processes. On the other hand, in our model for $m_{Z^{\prime}} \sim \mathrm{TeV}$ the contact interactions read

$$
\mathcal{L}_{\text {eff }}=-(1+\delta) \frac{g_{H}^{2}}{m_{Z^{\prime}}^{2}} \bar{e}_{R} \gamma_{\mu} e_{R} \bar{f}_{R} \gamma^{\mu} f_{R} .
$$

It turns out the strongest constraint arises from $e_{L}^{+} e_{R}^{-} \rightarrow e_{L}^{+} e_{R}^{-}$with $\Lambda=8.9 \mathrm{TeV}$ and $\eta=-1[55]$, which implies

$$
\frac{g_{H}}{m_{Z^{\prime}}} \lesssim \frac{0.2}{\mathrm{TeV}} \text { and } v_{\Phi} \gtrsim 10 \mathrm{TeV}
$$

In figure 3 , in the plane of $g_{H}$ and $m_{Z^{\prime}}$, we show the three constraints: the $Z-Z^{\prime}$ mixing by the blue dotted line, the heavy narrow dilepton resonance searches from CMS [62] in red and the LEP bounds on the electron-positron scattering cross-section of $e^{+} e^{-} \rightarrow e^{+} e^{-}[55]$ in black, where the region above each of the lines is excluded. The direct searches are dominant for most of the parameter space of interest, while the LEP constraint becomes most stringent toward the high-mass region. From this figure, we infer that for $0.1 \lesssim$ $g_{H} \lesssim 1$, the $Z^{\prime}$ mass has to be of order $\mathcal{O}(\mathrm{TeV})$. Note that $m_{Z^{\prime}} \approx g_{H} v_{\Phi} / 2$ and it implies $v_{\Phi} \sim 30 \mathrm{TeV}$ for $m_{Z^{\prime}} \sim 1.5 \mathrm{TeV}$ but $v_{\Phi}$ can be $10 \mathrm{TeV}$ for $m_{Z^{\prime}} \gtrsim 2.75 \mathrm{TeV}$.

\subsection{Constraints from the $125 \mathrm{GeV}$ SM-like Higgs}

We begin with the SM Higgs boson $h_{1}$ partial decay widths into SM fermions. Based on the Yukawa couplings in eqs. (2.9), (2.10) and (2.11), only the flavor eigenstate $H_{1}$ couples to two SM fermions. Thus, the coupling of the mass eigenstate $h_{1}$ to the SM fermions is rescaled by $O_{11}$, making the decay widths universally reduced by $O_{11}^{2}$ compared to the SM, which is different from generic $2 \mathrm{HDMs}$.

For the tree-level (off-shell) $h_{1} \rightarrow W^{+} W^{-}$, since the SM $W$ bosons do not mix with additional $\mathrm{SU}(2)_{H}$ (and $\left.\mathrm{U}(1)_{X}\right)$ gauge bosons and $\phi_{2}$ and $\delta_{3}$ are singlets under the $\mathrm{SU}(2)_{L}$ gauge group, the partial decay width is also suppressed by $O_{11}^{2}$. On the other hand, $h_{1} \rightarrow Z Z$ receives additional contributions from the $Z-Z^{\prime}$ mixing and thus the $\delta_{3}$ and 

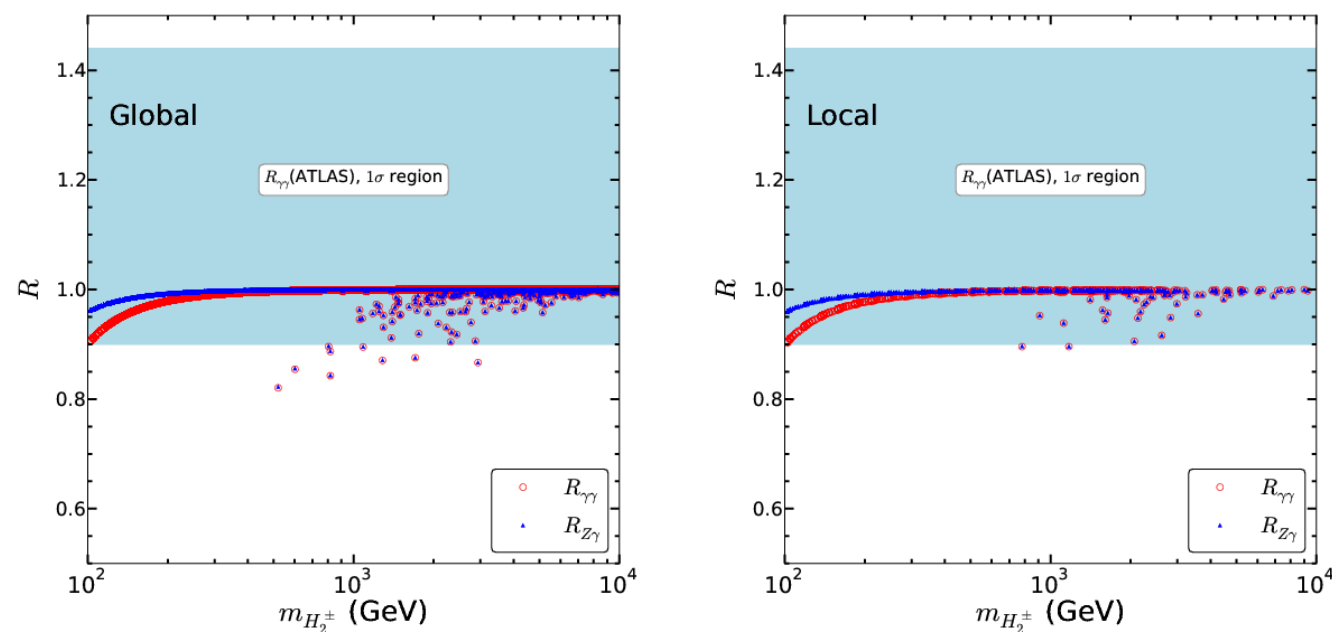

Figure 4. Predictions of $h \rightarrow \gamma \gamma$ and $h \rightarrow Z \gamma$ in this model. Due to the fact the $\left|O_{11}\right| \sim 1$ and $\lambda_{H}$ is always positive, $R_{\gamma \gamma}$ is often less than the SM prediction while $R_{Z \gamma}$ ranges from 0.9 to 1 , given the ATLAS and CMS measurements on $R_{\gamma \gamma}:$ : $1.17 \pm 0.27$ (ATLAS [64]) and $1.13 \pm 0.24$ (CMS [65]). Only ATLAS result is shown which completely covers the CMS $1 \sigma$ confidence region. The left (right) panel is for Global (Local) scenario.

$\phi_{2}$ components charged under $\mathrm{SU}(2)_{H}$ will also contribute. The mixing, however, are constrained to be small $\left(\lesssim 10^{-3}\right)$ by the EWPT data and LEP measurement on the electronpositron scattering cross-section as discussed in previous subsection. We will not consider the mixing effect here and the decay width is simply reduced by $O_{11}^{2}$ identical to other tree-level decay channels.

Now, we are in a position to explore the Higgs radiative decay rates into two photons and one $Z$ boson and one photon, normalized to the SM predictions. For convenience, we define $R_{X X}$ to be the production cross section of an SM Higgs boson decaying to $X X$ divided by the SM expectation as follows:

$$
R_{X X} \equiv \frac{\sigma\left(p p \rightarrow h_{1}\right)}{\sigma_{\mathrm{SM}}\left(p p \rightarrow h_{1}\right)} \frac{\operatorname{Br}\left(h_{1} \rightarrow X X\right)}{\operatorname{Br}_{\mathrm{SM}}\left(h_{1} \rightarrow X X\right)} .
$$

Note that first additional heavy colored fermions do not modify the Higgs boson production cross section, especially via $g g \rightarrow h_{1}$, because the $\mathrm{SU}(2)_{H}$ symmetry forbids the coupling of $h_{1} \bar{f} f$, where $f$ is the heavy fermion. Second, in order to avoid the observed Higgs invisible decay constraints in the vector boson fusion production mode: $\operatorname{Br}\left(h_{1} \rightarrow\right.$ invisible $)<$ $0.29[66,67]$, we constrain ourself to the region of $2 m_{D}>m_{h}$ so that the Higgs decay channels are the same as in the SM. As a consequence, eq. (4.11) becomes,

$$
R_{\gamma \gamma}=\frac{\Gamma\left(h_{1} \rightarrow \gamma \gamma\right)}{\Gamma_{\mathrm{SM}}\left(h_{1} \rightarrow \gamma \gamma\right)}, \quad R_{Z \gamma}=\frac{\Gamma\left(h_{1} \rightarrow \gamma Z\right)}{\Gamma_{\mathrm{SM}}\left(h_{1} \rightarrow \gamma \gamma\right)},
$$

similar to the situation of IHDM.

As mentioned before due to the existence of $\mathrm{SU}(2)_{H}$, there are no terms involving one SM Higgs boson and two heavy fermions, which are not $\mathrm{SU}(2)_{H}$ invariant. Therefore, the 
only new contributions to $h_{1} \rightarrow \gamma \gamma$ and $h_{1} \rightarrow Z \gamma$ arise from the heavy charged Higgs boson, $H_{2}^{ \pm}$. In addition, $h_{1}$ consists of $\delta_{3}$ and $\phi_{2}$ apart from $H_{1}^{0}$. With the quartic interactions $2 \lambda_{H} H_{2}^{\dagger} H_{2} H_{1}^{\dagger} H_{1}+\lambda_{H \Delta} H_{2}^{\dagger} H_{2} \Delta_{3}^{2} / 2+\lambda_{H \Phi} H_{2}^{\dagger} H_{2} \Phi_{2}^{*} \Phi_{2}$, there are in total three contributions to the $H_{2}^{ \pm}$-loop diagram. The Higgs decay widths into $\gamma \gamma$ and $Z \gamma$ including new scalars can be found in refs. [68-71] and they are collected in appendix B for convenience.

The results of $h \rightarrow \gamma \gamma$ (red circle) and $h \rightarrow Z \gamma$ (blue square) are presented in figure 4 for both the Global minimum case (left) and additional points which having the correct Higgs mass only at Local minimum (right). All the scatter points were selected from our grid scan described in subsection 4.1. It clearly shows that the mass of the heavy charged Higgs has to be larger than $100 \mathrm{GeV}$ in order to satisfy the LHC measurements on $h \rightarrow \gamma \gamma[64,65]$ while the corresponding $h \rightarrow Z \gamma$ ranges from 0.9 to 1 . Unlike normal IHDM where $\lambda_{3}$ in $\lambda_{3}\left|H_{1}\right|^{2}\left|H_{2}\right|^{2}$ can be either positive or negative, in this model we have $\lambda_{H}\left(\left|H_{1}\right|^{2}+\left|H_{2}\right|^{2}\right)^{2}$, where $\lambda_{H}$ as a quartic coupling has to be positive to ensure the potential is bounded from below. It implies that for $\left|O_{11}\right|^{2}$ being very close to 1 like the benchmark point $\mathrm{C}$ in table $2, h_{1}$ is mostly $h$, the neutral component in $H_{1}$, and $R_{\gamma \gamma}$ is determined by $\lambda_{H}$. In this case, $R_{\gamma \gamma}$, corresponding to the red curve, is always smaller than 1 in the region of interest where $m_{H_{2}^{ \pm}}>80 \mathrm{GeV}$ (due to the LEP constraints on the charged Higgs in the context of IHDM [72]), while $R_{Z \gamma}$ denoted by the blue curve also has values below the SM prediction as well.

In contrast, there are some points such as the benchmark point A where the contributions from $\phi_{2}$ and $\delta_{3}$ are significant, they manifest in figure 4 as scatter points away from the lines but also feature $R_{\gamma \gamma}, R_{Z \gamma}<1$.

\subsection{Dark matter stability}

In G2HDM, although there is no residual (discrete) symmetry left from $\mathrm{SU}(2)_{H}$ symmetry breaking, the lightest particle among the heavy $\mathrm{SU}(2)_{H}$ fermions $\left(u^{H}, d^{H}, e^{H}, \nu^{H}\right)$, the $\mathrm{SU}(2)_{H}$ gauge boson $W^{\prime}$ (but not $Z^{\prime}$ because of $Z-Z^{\prime}$ mixing) and the second heaviest eigenstate in the mass matrix of eq. $(3.8)^{4}$ is stable due to the gauge symmetry and the Lorentz invariance for the given particle content. In this work, we focus on the case of the second heaviest eigenstate in the mass matrix of eq. (3.8), a linear combination of $H_{2}^{0 *}, \Delta_{p}$ and $G_{H}^{p}$, being DM by assuming the $\mathrm{SU}(2)_{H}$ fermions and $W^{\prime}$ are heavier than it.

At tree level, it is clear that all renormalizable interactions always have even powers of the potential DM candidates in the set $D \equiv\left\{u^{H}, d^{H}, e^{H}, \nu^{H}, W^{\prime}, H_{2}^{0 *}, \Delta_{p}, G_{H}^{p}\right\}$. It implies they always appear in pairs and will not decay solely into SM particles. In other words, the decay of a particle in $D$ must be accompanied by the production of another particle in $D$, rendering the lightest one among $D$ stable.

Beyond the renormalizable level, one may worry that radiative corrections involving DM will create non-renormalizable interactions, which can be portrayed by high order effective operators, and lead to the DM decay. Assume the DM particle can decay into solely SM particles via certain higher dimensional operators, which conserve the gauge symmetry but spontaneously broken by the vevs of $H_{1}, \Phi_{H}, \Delta_{H}$. The SM particles refer

\footnotetext{
${ }^{4}$ The lightest one being the Goldstone boson absorbed by $W^{\prime}$.
} 
to SM fermions, Higgs boson $h$ and $\phi_{2}$ (which mixes with $h$ ). ${ }^{5}$ The operators, involving one DM particle and the decay products, are required to conserve the gauge symmetry. We here focus on 4 quantum numbers: $\mathrm{SU}(2)_{L}$ and $\mathrm{SU}(2)_{H}$ isospin, together with $\mathrm{U}(1)_{Y}$ and $\mathrm{U}(1)_{X}$ charge. $\mathrm{DM}$ is a linear combination of three flavour states: $H_{2}^{0 *}, \Delta_{p}$ and $G_{H}^{p}$. First, we study operators with $H_{2}^{0 *}$ decaying leptonically into $n_{1} \nu_{L} \mathrm{~s}, n_{2} e_{L} \mathrm{~s}, n_{3} \nu_{R} \mathrm{~s}, n_{4}$ $e_{R} \mathrm{~s}, n_{5} h \mathrm{~s}$ and $n_{6} \phi_{2} \mathrm{~s} .{ }^{6}$ One has, in terms of the quantum numbers $\left(I_{3}^{H}, I_{3}, Y, X\right)$,

$$
\begin{aligned}
(1 / 2,1 / 2,-1 / 2,-1)= & n_{1} *(0,1 / 2,-1 / 2,0)+n_{2} *(0,-1 / 2,-1 / 2,0) \\
& +n_{3} *(1 / 2,0,0,1)+n_{4} *(-1 / 2,0,-1,-1) \\
& +n_{5} *(1 / 2,-(1 / 2), 1 / 2,1)+n_{6} *(-1 / 2,0,0,1),
\end{aligned}
$$

which gives

$$
n_{3}=1-n_{1}, n_{4}=-n_{2}, n_{5}=-1+n_{1}-n_{2}, n_{6}=-1 .
$$

This implies the number of net fermions (fermions minus anti-fermions) is $n_{1}+n_{2}+n_{3}+n_{4}=$ 1 , where positive and negative $n_{i}$ correspond to fermion and anti-fermion respectively. In other words, if the number of fermions is odd, the number of anti-fermions must be even; and vice versa. Clearly, this implies Lorentz violation since the total fermion number (fermions plus anti-fermions) is also odd. Therefore, the flavor state $H_{2}^{0 *}$ can not decay solely into SM particles. It is straightforward to show the conclusion applies to $\Delta_{p}$ and $G_{H}^{p}$ as well. As a result, the DM candidate, a superposition of $H_{2}^{0 *}, \Delta_{p}$ and $G_{H}^{p}$, is stable as long as it is the lightest one among the potential dark matter candidates $D$.

Before moving to the DM relic density computation, we would like to comment on effective operators with an odd number of $D$, like three $D$ s or more. It is not possible that this type of operators, invariant under the gauge symmetry, will induce operators involving only one DM particle, by connecting an even number of $D$ s into loop. It is because those operators linear in $D$ violate either the gauge symmetry or Lorentz invariance and hence can never be (radiatively) generated from operators obeying the symmetries. After all, the procedure of reduction on the power of $D$, i.e., closing loops with proper vev insertions (which amounts to adding Yukawa terms), also complies with the gauge and Lorentz symmetry.

\subsection{Dark matter relic density}

We now show this model can reproduce the correct DM relic density. As mentioned above, the DM particle will be a linear combination of $G_{H}^{p}, \Delta_{p}$ and $H_{2}^{0 *}$. Thus (co)-annihilation channels are relevant if their masses are nearly degenerated and the analysis can be quite involved. In this work, we constrain ourselves in the simple limit where the G2HDM becomes IHDM, in which case the computation of DM relic density has been implemented in the software package micrOMEGAs [73, 74]. For a recent detailed analysis of IHDM, see ref. [10] and references therein. In other words, we are working in the scenario that the

\footnotetext{
${ }^{5}$ The SM gauge bosons are not included since they decay into SM fermions, while inclusion of $\delta_{3}$ will not change the conclusion because it carries zero charge in term of the quantum numbers in consideration.

${ }^{6}$ The conclusion remains the same if quarks are also included.
} 
DM being mostly $H_{2}^{0}$ and the SM Higgs boson $h$ has a minute mixing with $\delta_{3}$ and $\phi_{2}$. The IHDM Higgs potential reads,

$$
\begin{aligned}
V_{\mathrm{IHDM}}= & \mu_{1}^{2}\left|H_{1}\right|^{2}+\mu_{2}^{2}\left|H_{2}\right|^{2}+\lambda_{1}\left|H_{1}\right|^{4}+\lambda_{2}\left|H_{2}\right|^{4}+\lambda_{3}\left|H_{1}\right|^{2}\left|H_{2}\right|^{2}+\lambda_{4}\left|H_{1}^{\dagger} H_{2}\right|^{2} \\
& +\frac{\lambda_{5}}{2}\left\{\left(H_{1}^{\dagger} H_{2}\right)^{2}+\text { h.c. }\right\},
\end{aligned}
$$

where $\lambda_{1}=\lambda_{2}=2 \lambda_{3}=\lambda_{H}$ with $\lambda_{4}=\lambda_{5}=0$ when compared to our model. It implies the degenerate mass spectrum for $H_{2}^{ \pm}$and $H_{2}^{0}$. The mass splitting, nonetheless, arises from the fact $H_{2}^{0}$ (DM particle) also receives a tiny contribution from $\Delta_{p}$ and $G_{H}^{p}$ as well as loop contributions. In the limit of IHDM the mass splitting is very small, making paramount (co)-annihilations, such as $H_{2}^{+} H_{2}^{-} \rightarrow W^{+} W^{-}, H_{2}^{0} H_{2}^{0} \rightarrow W^{+} W^{-},\left(H_{2}^{+} H_{2}^{-}, H_{2}^{0} H_{2}^{0}\right) \rightarrow Z Z$, $H_{2}^{ \pm} H_{2}^{0} \rightarrow W^{ \pm} \gamma$, and thus the DM relic density is mostly determined by these channels.

Besides, from eq. (4.15), $\lambda_{H}$ is seemingly fixed by the Higgs mass, $\lambda_{1}=m_{h}^{2} / 2 v^{2} \sim 0.13$, a distinctive feature of IHDM. In G2HDM, however, the value of $\lambda_{H}$ can deviate from 0.13 due to the mixing among $h, \delta_{3}$ and $\phi_{2}$ as shown in figure 1, where the red curve corresponds to the Higgs boson mass of $125 \mathrm{GeV}$ with $\lambda_{H}$ varying from 1.2 to 1.9 as a function of $M_{H \Delta}$, which controls the scalar mixing. To simulate G2HDM but still stay close to the IHDM limit, we will treat $\lambda_{H}$ as a free parameter in the analysis and independent of the SM Higgs mass.

We note that micrOMEGAs requires five input parameters for IHDM: SM Higgs mass (fixed to be $125 \mathrm{GeV}$ ), $H_{2}^{ \pm}$mass, $H_{2}^{0}$ mass (including both CP-even and odd components which are degenerate in our model $), \lambda_{2}\left(=\lambda_{H}\right)$ and $\left(\lambda_{3}+\lambda_{4}-\lambda_{5}\right) / 2\left(\approx \lambda_{H}\right)$. It implies that only two of them are independent in G2HDM, which can be chosen to be $m_{\mathrm{DM}}$ and $\lambda_{H}$. Strictly speaking, $m_{\mathrm{DM}}$ is a function of parameters such as $M_{\Phi \Delta}, M_{H \Delta}, v_{\Phi}$, $v_{\Delta}$, etc., since it is one of the eigenvalues in the mass matrix of eq. (3.8). In this analysis, we stick to the scan range of the parameters displayed in eq. (4.1) with slight modification as follows

$$
\begin{array}{r}
0.12 \leq \lambda_{H} \leq \quad 0.2, \\
0.8 \leq M_{\Phi \Delta} / M_{H \Delta} \leq 1.0,
\end{array}
$$

and also demand the mixing (denoted by $\mathcal{O}_{D}$ ) between $H_{2}^{0}$ and the other scalars to be less than $1 \%$. In the exact IHDM limit, $\mathcal{O}_{D}$ should be 1 . Besides, the decay time of $H_{2}^{ \pm}$ into $H_{2}^{0}$ plus an electron and an electron neutrino is required to be much shorter than one second in order not to spoil the Big Bang nucleosynthesis.

Our result is presented in figure 5. For a given $\lambda_{H}$, there exists an upper bound on the DM mass, above which the DM density surpasses the observed one, as shown in the left panel of figure 5 . The brown band in the plot corresponds to the DM relic density of $0.1<\Omega h^{2}<0.12$ while the yellow band refers to $\Omega h^{2}<0.1$. In the right panel of figure 5 , we show how well the IHDM limit can be reached in the parameter space of $M_{\Phi \Delta} / M_{H \Delta}$ versus $v_{\Delta} / v_{\Phi}$, where the red band corresponds to $0.8<\mathcal{O}_{D}^{2}<0.9$, the green band refers to $0.9<\mathcal{O}_{D}^{2}<0.99$ (green) and while the light blue area represents $0.99<\mathcal{O}_{D}^{2}$. One can easily see the IHDM limit $\left(\mathcal{O}_{D}^{2} \sim 1\right)$ can be attained with $M_{\Phi \Delta} \lesssim M_{H \Delta}$. For $M_{\Phi \Delta}>M_{H \Delta}$, we 

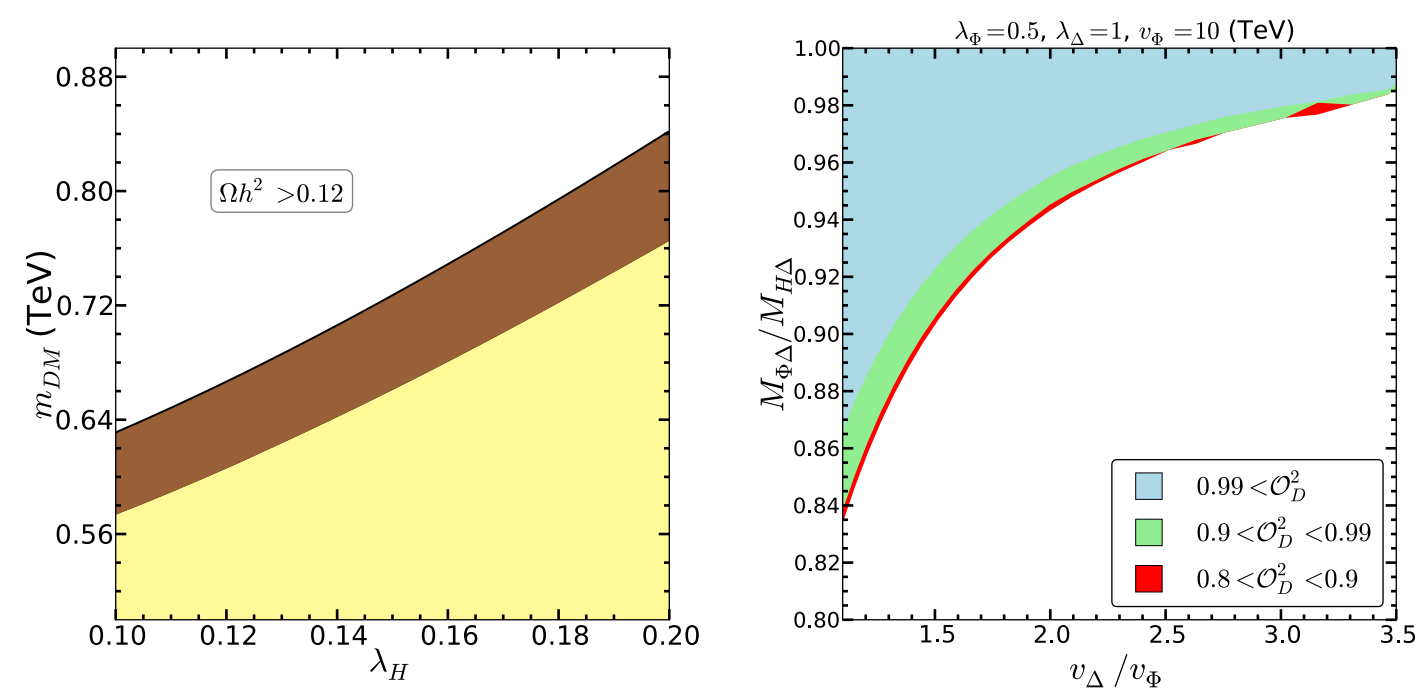

Figure 5. Left: the contour of relic density on $\left(\lambda_{H}, m_{D M}\right)$ plane. The upper brown region is with the relic density between 0.1 and 0.12 but the lower yellow region is the relic density less than 0.1. Right: accepted DM mass region projected on $\left(v_{\Delta} / v_{\Phi}, M_{\Phi \Delta} / M_{H \Delta}\right)$ plane. The red, green and light blue regions present the DM inert Higgs fraction $0.8<\mathcal{O}_{D}^{2}<0.9,0.9<\mathcal{O}_{D}^{2}<0.99$ and $0.99<\mathcal{O}_{D}^{2}$, respectively. See the text for detail of the analysis.

have $m_{H_{2}^{ \pm}}<m_{H_{2}^{0}}$, implying $H_{2}^{0}$ can not be DM anymore. Finally, we would like to point out that the allowed parameter space may increase significantly once the small $H_{2}^{0}$ mixing constraint is removed and other channels comprising $G_{H}^{p}$ and $\Delta_{p}$ are considered.

It is worthwhile to mention that not only $H_{2}^{0}$ but also $\nu^{H}$ (right-handed neutrino's $\mathrm{SU}(2)_{H}$ partner) can be the DM candidate in G2HDM, whose stability can be proved similarly based on the gauge and Lorentz invariance. If this is the case, then there exists an intriguing connection between DM phenomenology and neutrino physics. We, however, will leave this possibility for future work.

\subsection{EWPT $-\Delta S, \Delta T$ and $\Delta U$}

In light of the existence of new scalars and fermions, ${ }^{7}$ one should be concerned about the EWPT, characterized by the oblique parameters $\Delta S, \Delta T$ and $\Delta U$ [75]. For scalar contributions, similar to the situation of DM relic density computation, a complete calculation in G2HDM can be quite convoluted. The situation, however, becomes much simpler when one goes to the IHDM limit as before, i.e., $H_{2}^{0}$ is virtually the mass eigenstate and the inert doublet $H_{2}$ is the only contribution to $\Delta S, \Delta T$ and $\Delta U$, since $\Phi_{H}$ and $\Delta_{H}$ are singlets under the SM gauge group. Analytic formulas for the oblique parameters can be found in ref. [8]. All of them will vanish if the mass of $H_{2}^{ \pm}$is equal to that of $H_{2}^{0}$ as guaranteed by the $\mathrm{SU}(2)_{L}$ invariance.

\footnotetext{
${ }^{7}$ For additional gauge bosons in the limit of $\mathrm{U}(1)_{X}$ being a global symmetry, the constrain considered above, $\sin \theta_{Z Z^{\prime}}<10^{-3}$, are actually derived from the electroweak precision data combined with collider bounds. As a consequence, we will not discuss their contributions again.
} 
On the other hand, the mass splitting stems from the $H_{2}^{0 *}$ mixing with $G_{H}^{p}$ and $\Delta_{p}$; therefore, in the limit of IHDM, the mass splitting is very small, implying a very small deviation from the SM predictions of the oblique parameters. We have numerically checked that all points with the correct DM relic abundance studied in the previous section have negligible $H_{2}$ contributions, of order less than $10^{-3}$, to $\Delta S, \Delta T$ and $\Delta U$.

Finally for the heavy fermions, due to the fact they are singlets under $\mathrm{SU}(2)_{L}$, the corresponding contributions will vanish according to the definition of oblique parameters [75]. Our model survives the challenge from the EWPT as long as one is able to suppress the extra scalar contributions, for instance, resorting to the IHDM limit or having cancellation among different contributions.

\section{Conclusions and outlook}

In this work, we propose a novel framework to embed two Higgs doublets, $H_{1}$ and $H_{2}$ into a doublet under a non-abelian gauge symmetry $\mathrm{SU}(2)_{H}$ and the $\mathrm{SU}(2)_{H}$ doublet is charged under an additional abelian group $\mathrm{U}(1)_{X}$. To give masses to additional gauge bosons, we introduce an $\mathrm{SU}(2)_{H}$ scalar triplet and doublet (singlets under the SM gauge group). The potential of the two Higgs doublets is as simple as the SM Higgs potential at the cost of additional terms involving the $\mathrm{SU}(2)_{H}$ triplet and doublet. The vev of the triplet triggers the spontaneous symmetry breaking of $\mathrm{SU}(2)_{L}$ by generating the vev for the first $\mathrm{SU}(2)_{L}$ Higgs doublet, identified as the SM Higgs doublet, while the second Higgs doublet does not obtain a vev and the neutral component could be the DM candidate, whose stability is guaranteed by the $\mathrm{SU}(2)_{H}$ symmetry and Lorentz invariance. Instead of investigating DM phenomenology, we have focused here on Higgs physics and mass spectra of new particles.

To ensure the model is anomaly-free and SM Yukawa couplings preserve the additional $\mathrm{SU}(2)_{H} \times \mathrm{U}(1)_{X}$ symmetry, we choose to place the SM right-handed fermions and new heavy right-handed fermions into $\mathrm{SU}(2)_{H}$ doublets while $\mathrm{SM} \mathrm{SU}(2)_{L}$ fermion doublets are singlets under $\mathrm{SU}(2)_{H}$. Moreover, the vev of the $\mathrm{SU}(2)_{H}$ scalar doublet can provide a mass to the new heavy fermions via new Yukawa couplings.

Different from the left-right symmetric model with a bi-doublet Higgs bosons, in which $W_{R}^{ \pm}$carries the electric charge, the corresponding $W^{\prime}$ bosons in this model that connects $H_{1}$ and $H_{2}$ are electrically neutral since $H_{1}$ and $H_{2}$ have the same SM gauge group charges. On the other hand, the corresponding $Z^{\prime}$ actually mixes with the SM $Z$ boson and the mixing are constrained by the EWPT data as well as collider searches on the $W^{ \pm}$final state. $Z^{\prime}$ itself is also confronted by the direct resonance searches based on dilepton or dijet channels, limiting the vev of the scalar doublet $\Phi$ to be of order $\mathcal{O}(\mathrm{TeV})$.

By virtue of the mixing between other neutral scalars and the neutral component of $H_{1}$, the SM Higgs boson is a linear combination of three scalars. So the SM Higgs boson treelevel couplings to the SM fermions and gauge bosons are universally rescaled by the mixing angle with respect to the SM results. We also check the $h \rightarrow \gamma \gamma$ decay rate normalized to the SM value and it depends not only on the mass of $H_{2}^{ \pm}$but also other mixing parameters. As a result, $H_{2}^{ \pm}$has to be heavier than $100 \mathrm{GeV}$ while the $h \rightarrow Z \gamma$ decay width is very close to the SM prediction. We also confirm that our model can reproduce the correct 
DM relic abundance and stays unscathed from the EWPT data in the limit of IHDM where DM is purely the second neutral Higgs $H_{2}^{0}$. Detailed and systematic study will be pursued elsewhere.

As an outlook, we briefly comment on collider signatures of this model, for which detailed analysis goes beyond the scope of this work and will be pursued in the future. Due to the $\mathrm{SU}(2)_{H}$ symmetry, searches for heavy particles are similar to those of SUSY partners of the SM particles with $R$-parity. In the case of $H_{2}^{0}$ being the DM candidate, one can have, for instance, $u_{R} \overline{u_{R}} \rightarrow W^{\prime p} W^{\prime m}$ via $t$-channel exchange of $u_{R}^{H}$, followed by $W^{\prime p} \rightarrow \overline{u_{R}} u_{R}^{H} \rightarrow \overline{u_{R}} H_{2}^{0} u_{L}$ and its complex conjugate, leading to 4 jets plus missing transverse energy. Therefore, searches on charginos or gauginos in the context of SUSY may also apply to this model. Furthermore, this model can also yield mono-jet or monophoton signatures: $u_{R} \overline{u_{R}} \rightarrow H_{2}^{0} H_{2}^{0}$ plus $\gamma$ or $g$ from the initial state radiation. Finally, the recent diboson excess observed by the ATLAS Collaboration [76] may be partially explained by $2 \mathrm{TeV} Z^{\prime}$ decays into $W^{+} W^{-}$via the $Z^{\prime}-Z$ mixing.

Phenomenology of G2HDM is quite rich. In this work we have only touched upon its surface. Many topics like constraints from vacuum stability as well as DM and neutrinos physics, collider implications, etc are worthwhile to be pursued further. We would like to return to some of these issues in the future.

\section{A Analytical expression for $v, v_{\Delta}$ and $v_{\Phi}$}

From eqs. (3.3) and (3.4), besides the trivial solutions of $v^{2}=v_{\Phi}^{2}=0$ one can deduce the following non-trivial expressions for $v^{2}$ and $v_{\Phi}^{2}$ respectively,

$$
\begin{aligned}
& v^{2}=\frac{\left(2 \lambda_{\Phi} \lambda_{H \Delta}-\lambda_{H \Phi} \lambda_{\Phi \Delta}\right) v_{\Delta}^{2}+\left(\lambda_{H \Phi} M_{\Phi \Delta}-2 \lambda_{\Phi} M_{H \Delta}\right) v_{\Delta}+2\left(2 \lambda_{\Phi} \mu_{H}^{2}-\lambda_{H \Phi} \mu_{\Phi}^{2}\right)}{\lambda_{H \Phi}^{2}-4 \lambda_{H} \lambda_{\Phi}}, \\
& v_{\Phi}^{2}=\frac{\left(2 \lambda_{H} \lambda_{\Phi \Delta}-\lambda_{H \Phi} \lambda_{H \Delta}\right) v_{\Delta}^{2}+\left(\lambda_{H \Phi} M_{H \Delta}-2 \lambda_{H} M_{\Phi \Delta}\right) v_{\Delta}+2\left(2 \lambda_{H} \mu_{\Phi}^{2}-\lambda_{H \Phi} \mu_{H}^{2}\right)}{\lambda_{H \Phi}^{2}-4 \lambda_{H} \lambda_{\Phi}} .
\end{aligned}
$$

Substituting the above expressions for $v^{2}$ and $v_{\Phi}^{2}$ into eq. (3.5) leads to the following cubic equation for $v_{\Delta}$ :

$$
v_{\Delta}^{3}+a_{2} v_{\Delta}^{2}+a_{1} v_{\Delta}+a_{0}=0
$$

where $a_{2}=C_{2} / C_{3}, a_{1}=C_{1} / C_{3}$ and $a_{0}=C_{0} / C_{3}$ with

$$
\begin{aligned}
C_{0}= & 2\left(\lambda_{H \Phi} M_{\Phi \Delta}-2 \lambda_{\Phi} M_{H \Delta}\right) \mu_{H}^{2}+2\left(\lambda_{H \Phi} M_{H \Delta}-2 \lambda_{H} M_{\Phi \Delta}\right) \mu_{\Phi}^{2}, \\
C_{1}= & 2\left[2\left(2 \lambda_{H \Delta} \lambda_{\Phi}-\lambda_{H \Phi} \lambda_{\Phi \Delta}\right) \mu_{H}^{2}+2\left(2 \lambda_{H} \lambda_{\Phi \Delta}-\lambda_{H \Delta} \lambda_{H \Phi}\right) \mu_{\Phi}^{2}\right. \\
& \left.+2\left(4 \lambda_{H} \lambda_{\Phi}-\lambda_{H \Phi}^{2}\right) \mu_{\Delta}^{2}+\lambda_{H} M_{\Phi \Delta}^{2}-\lambda_{H \Phi} M_{H \Delta} M_{\Phi \Delta}+\lambda_{\Phi} M_{H \Delta}^{2}\right], \\
C_{2}= & 3\left[\left(\lambda_{H} \lambda_{H \Phi}-2 \lambda_{H} \lambda_{\Phi \Delta}\right) M_{\Phi \Delta}+\left(\lambda_{H \Phi} \lambda_{\Phi \Delta}-2 \lambda_{H \Delta} \lambda_{\Phi}\right) M_{H \Delta}\right], \\
C_{3}= & 4\left[\lambda_{H}\left(\lambda_{\Phi \Delta}^{2}-4 \lambda_{\Delta} \lambda_{\Phi}\right)-\lambda_{H \Delta} \lambda_{H \Phi} \lambda_{\Phi \Delta}+\lambda_{H \Delta}^{2} \lambda_{\Phi}+\lambda_{\Delta} \lambda_{H \Phi}^{2}\right] .
\end{aligned}
$$


The three roots of cubic equation like eq. (A.3) are well-known since the middle of $16^{\text {th }}$ century

$$
\begin{aligned}
& v_{\Delta 1}=-\frac{1}{3} a_{2}+(S+T), \\
& v_{\Delta 2}=-\frac{1}{3} a_{2}-\frac{1}{2}(S+T)+\frac{1}{2} i \sqrt{3}(S-T), \\
& v_{\Delta 3}=-\frac{1}{3} a_{2}-\frac{1}{2}(S+T)-\frac{1}{2} i \sqrt{3}(S-T),
\end{aligned}
$$

where

$$
\begin{aligned}
S & \equiv \sqrt[3]{R+\sqrt{D}}, \\
T & \equiv \sqrt[3]{R-\sqrt{D}}, \\
D & \equiv Q^{3}+R^{2}
\end{aligned}
$$

with

$$
\begin{aligned}
& Q \equiv \frac{3 a_{1}-a_{2}^{2}}{9} \\
& R \equiv \frac{9 a_{1} a_{2}-27 a_{0}-2 a_{2}^{3}}{54} .
\end{aligned}
$$

\section{B Decay width of SM Higgs to $\gamma \gamma$ and $Z \gamma$}

Below we summarize the results for the decay width of SM Higgs to $\gamma \gamma$ and $\gamma Z$ [68-71], including the mixing among $h, \delta_{3}$ and $\phi_{2}$ characterized by the orthogonal matrix $O$, i.e., $\left(h, \delta_{3}, \phi_{2}\right)^{T}=O \cdot\left(h_{1}, h_{2}, h_{3}\right)^{T}$. In general one should include the mixing effects among $Z$ and $Z^{\prime}$ (and perhaps $Z^{\prime \prime}$ ) as well. As shown in section IV, these mixings are constrained to be quite small and we will ignore them here.

- Taking into account $H_{2}^{ \pm}$contributions, the partial width of $h_{1} \rightarrow \gamma \gamma$ is

$$
\Gamma\left(h_{1} \rightarrow \gamma \gamma\right)=\frac{G_{F} \alpha^{2} m_{h_{1}}^{3} O_{11}^{2}}{128 \sqrt{2} \pi^{3}}\left|\mathcal{C}_{h} \frac{\lambda_{H} v^{2}}{m_{H_{2}^{ \pm}}^{2}} A_{0}^{\gamma \gamma}\left(\tau_{H_{2}^{ \pm}}\right)+A_{1}^{\gamma \gamma}\left(\tau_{W}\right)+\sum_{f} N_{c} Q_{f}^{2} A_{1 / 2}^{\gamma \gamma}\left(\tau_{f}\right)\right|^{2}
$$

with

$$
\mathcal{C}_{h}=1-\frac{O_{21}}{O_{11}} \frac{2 \lambda_{H \Delta} v_{\Delta}+M_{H \Delta}}{4 \lambda_{H} v}+\frac{O_{31}}{O_{11}} \frac{\lambda_{H \Phi} v_{\Phi}}{2 \lambda_{H} v} .
$$

The form factors for spins $0, \frac{1}{2}$ and 1 particles are given by

$$
\begin{aligned}
A_{0}^{\gamma \gamma}(\tau) & =-[\tau-f(\tau)] \tau^{-2}, \\
A_{1 / 2}^{\gamma \gamma}(\tau) & =2[\tau+(\tau-1) f(\tau)] \tau^{-2}, \\
A_{1}^{\gamma \gamma}(\tau) & =-\left[2 \tau^{2}+3 \tau+3(2 \tau-1) f(\tau)\right] \tau^{-2},
\end{aligned}
$$


with the function $f(\tau)$ defined by

$$
f(\tau)= \begin{cases}\arcsin ^{2} \sqrt{\tau} & , \text { for } \tau \leq 1 \\ -\frac{1}{4}\left[\log \frac{1+\sqrt{1-\tau^{-1}}}{1-\sqrt{1-\tau^{-1}}}-i \pi\right]^{2} & , \text { for } \tau>1 .\end{cases}
$$

The parameters $\tau_{i}=m_{h_{1}}^{2} / 4 m_{i}^{2}$ with $i=H_{2}^{ \pm}, f, W^{ \pm}$are related to the corresponding masses of the heavy particles in the loops.

- Including the $H_{2}^{ \pm}$contribution, we have

$$
\begin{aligned}
\Gamma\left(h_{1} \rightarrow Z \gamma\right)= & \frac{G_{F}^{2} m_{W}^{2} \alpha m_{h_{1}}^{3} O_{11}^{2}}{64 \pi^{4}}\left(1-\frac{m_{Z}^{2}}{m_{h_{1}}^{2}}\right)^{3} \times \\
& \times \mid-\mathcal{C}_{h} \frac{\lambda_{H} v^{2}}{m_{H_{2}^{ \pm}}^{2}} v_{H_{2}^{ \pm}} A_{0}^{Z \gamma}\left(\tau_{H_{2}^{ \pm}}, \lambda_{H_{2}^{ \pm}}\right)+A_{1}^{Z \gamma}\left(\tau_{W}, \lambda_{W}\right) \\
& +\left.\sum_{f} N_{c} \frac{Q_{f} \hat{v}_{f}}{c_{W}} A_{1 / 2}^{Z \gamma}\left(\tau_{f}, \lambda_{f}\right)\right|^{2},
\end{aligned}
$$

with $v_{H_{2}^{ \pm}}=\left(2 c_{W}^{2}-1\right) / c_{W}, \hat{v}_{f}=2 I_{f}^{3}-4 Q_{f} s_{W}^{2}, \tau_{i}=4 m_{i}^{2} / m_{h_{1}}^{2}$ and $\lambda_{i}=4 m_{i}^{2} / m_{Z}^{2}$. The loop functions are

$$
\begin{aligned}
A_{0}^{Z \gamma}\left(\tau_{H^{ \pm}}, \lambda_{H^{ \pm}}\right) & =I_{1}\left(\tau_{H^{ \pm}}, \lambda_{H^{ \pm}}\right), \\
A_{1 / 2}^{Z \gamma}(\tau, \lambda) & =\left[I_{1}(\tau, \lambda)-I_{2}(\tau, \lambda)\right], \\
A_{1}^{Z \gamma}(\tau, \lambda) & =c_{W}\left\{4\left(3-\frac{s_{W}^{2}}{c_{W}^{2}}\right) I_{2}(\tau, \lambda)+\left[\left(1+\frac{2}{\tau}\right) \frac{s_{W}^{2}}{c_{W}^{2}}-\left(5+\frac{2}{\tau}\right)\right] I_{1}(\tau, \lambda)\right\},
\end{aligned}
$$

where $I_{1}$ and $I_{2}$ are defined as

$$
\begin{aligned}
& I_{1}(\tau, \lambda)=\frac{\tau \lambda}{2(\tau-\lambda)}+\frac{\tau^{2} \lambda^{2}}{2(\tau-\lambda)^{2}}\left[f\left(\tau^{-1}\right)-f\left(\lambda^{-1}\right)\right]+\frac{\tau^{2} \lambda}{(\tau-\lambda)^{2}}\left[g\left(\tau^{-1}\right)-g\left(\lambda^{-1}\right)\right], \\
& I_{2}(\tau, \lambda)=-\frac{\tau \lambda}{2(\tau-\lambda)}\left[f\left(\tau^{-1}\right)-f\left(\lambda^{-1}\right)\right],
\end{aligned}
$$

with the function $f(\tau)$ defined in eq. (B.4) and the function $g(\tau)$ can be expressed as

$$
g(\tau)= \begin{cases}\sqrt{\tau^{-1}-1} \arcsin \sqrt{\tau} & , \text { for } \tau \geq 1 ; \\ \frac{\sqrt{1-\tau^{-1}}}{2}\left[\log \frac{1+\sqrt{1-\tau^{-1}}}{1-\sqrt{1-\tau^{-1}}}-i \pi\right], & \text { for } \tau<1 .\end{cases}
$$

The corresponding SM rates $\Gamma_{\mathrm{SM}}\left(h_{1} \rightarrow \gamma \gamma\right)$ and $\Gamma_{\mathrm{SM}}\left(h_{1} \rightarrow Z \gamma\right)$ can be obtained by omitting the $H_{2}^{ \pm}$contribution and setting $O_{11}=1$ in eqs. (B.1) and (B.5). 


\section{Acknowledgments}

The authors would like to thank A. Arhrib, F. Deppisch, M. Fukugita, J. Harz and T. Yanagida for useful discussions. WCH is grateful for the hospitality of IOP Academia Sinica and NCTS in Taiwan and HEP group at Northwestern University where part of this work was carried out. TCY is grateful for the hospitality of IPMU where this project was completed. This work is supported in part by the Ministry of Science and Technology (MoST) of Taiwan under grant numbers 101-2112-M-001-005-MY3 and 104-2112-M-001001-MY3 (TCY), the London Centre for Terauniverse Studies (LCTS) using funding from the European Research Council via the Advanced Investigator Grant 267352 (WCH), DGF Grant No. PA 803/10-1 (WCH), and the World Premier International Research Center Initiative (WPI), MEXT, Japan (YST).

Open Access. This article is distributed under the terms of the Creative Commons Attribution License (CC-BY 4.0), which permits any use, distribution and reproduction in any medium, provided the original author(s) and source are credited.

\section{References}

[1] ATLAS collaboration, Observation of a new particle in the search for the standard model Higgs boson with the ATLAS detector at the LHC, Phys. Lett. B 716 (2012) 1 [arXiv: 1207.7214] [INSPIRE].

[2] CMS collaboration, Observation of a new boson at a mass of $125 \mathrm{GeV}$ with the CMS experiment at the LHC, Phys. Lett. B 716 (2012) 30 [arXiv:1207.7235] [INSPIRE].

[3] V. Silveira and A. Zee, Scalar phantoms, Phys. Lett. B 161 (1985) 136 [InSPIRE].

[4] J. McDonald, Gauge singlet scalars as cold dark matter, Phys. Rev. D 50 (1994) 3637 [hep-ph/0702143] [INSPIRE].

[5] C.P. Burgess, M. Pospelov and T. ter Veldhuis, The minimal model of nonbaryonic dark matter: a singlet scalar, Nucl. Phys. B 619 (2001) 709 [hep-ph/0011335] [INSPIRE].

[6] N.G. Deshpande and E. Ma, Pattern of symmetry breaking with two Higgs doublets, Phys. Rev. D 18 (1978) 2574 [INSPIRE].

[7] E. Ma, Verifiable radiative seesaw mechanism of neutrino mass and dark matter, Phys. Rev. D 73 (2006) 077301 [hep-ph/0601225] [INSPIRE].

[8] R. Barbieri, L.J. Hall and V.S. Rychkov, Improved naturalness with a heavy Higgs: an alternative road to LHC physics, Phys. Rev. D 74 (2006) 015007 [hep-ph/0603188] [INSPIRE].

[9] L. Lopez Honorez, E. Nezri, J.F. Oliver and M.H.G. Tytgat, The inert doublet model: an archetype for dark matter, JCAP 02 (2007) 028 [hep-ph/0612275] [INSPIRE].

[10] A. Arhrib, Y.-L.S. Tsai, Q. Yuan and T.-C. Yuan, An updated analysis of inert Higgs doublet model in light of the recent results from LUX, PLANCK, AMS-02 and LHC, JCAP 06 (2014) 030 [arXiv:1310.0358] [INSPIRE].

[11] M.B. Gavela, P. Hernández, J. Orloff and O. Pene, Standard model CP-violation and baryon asymmetry, Mod. Phys. Lett. A 9 (1994) 795 [hep-ph/9312215] [INSPIRE]. 
[12] P. Huet and E. Sather, Electroweak baryogenesis and standard model CP-violation, Phys. Rev. D 51 (1995) 379 [hep-ph/9404302] [INSPIRE].

[13] M.B. Gavela, P. Hernández, J. Orloff, O. Pene and C. Quimbay, Standard model CP-violation and baryon asymmetry. Part 2: finite temperature, Nucl. Phys. B 430 (1994) 382 [hep-ph/9406289] [INSPIRE].

[14] A.I. Bochkarev and M.E. Shaposhnikov, Electroweak production of baryon asymmetry and upper bounds on the Higgs and top masses, Mod. Phys. Lett. A 2 (1987) 417 [INSPIRE].

[15] K. Kajantie, M. Laine, K. Rummukainen and M.E. Shaposhnikov, The electroweak phase transition: a nonperturbative analysis, Nucl. Phys. B 466 (1996) 189 [hep-lat/9510020] [INSPIRE].

[16] A.I. Bochkarev, S.V. Kuzmin and M.E. Shaposhnikov, Electroweak baryogenesis and the Higgs boson mass problem, Phys. Lett. B 244 (1990) 275 [INSPIRE].

[17] A.I. Bochkarev, S.V. Kuzmin and M.E. Shaposhnikov, On the model dependence of the cosmological upper bound on the Higgs boson and top quark masses, Phys. Rev. D 43 (1991) 369 [INSPIRE].

[18] N. Turok and J. Zadrozny, Electroweak baryogenesis in the two doublet model, Nucl. Phys. B 358 (1991) 471 [INSPIRE].

[19] A.G. Cohen, D.B. Kaplan and A.E. Nelson, Spontaneous baryogenesis at the weak phase transition, Phys. Lett. B 263 (1991) 86 [INSPIRE].

[20] A.E. Nelson, D.B. Kaplan and A.G. Cohen, Why there is something rather than nothing: matter from weak interactions, Nucl. Phys. B 373 (1992) 453 [INSPIRE].

[21] G.C. Branco, P.M. Ferreira, L. Lavoura, M.N. Rebelo, M. Sher and J.P. Silva, Theory and phenomenology of two-Higgs-doublet models, Phys. Rept. 516 (2012) 1 [arXiv:1106.0034] [INSPIRE].

[22] H.E. Haber, G.L. Kane and T. Sterling, The fermion mass scale and possible effects of Higgs bosons on experimental observables, Nucl. Phys. B 161 (1979) 493 [inSPIRE].

[23] L.J. Hall and M.B. Wise, Flavor changing Higgs-boson couplings, Nucl. Phys. B 187 (1981) 397 [INSPIRE].

[24] J.F. Donoghue and L.F. Li, Properties of charged Higgs bosons, Phys. Rev. D 19 (1979) 945 [INSPIRE].

[25] V.D. Barger, J.L. Hewett and R.J.N. Phillips, New constraints on the charged Higgs sector in two Higgs doublet models, Phys. Rev. D 41 (1990) 3421 [INSPIRE].

[26] A.G. Akeroyd and W.J. Stirling, Light charged Higgs scalars at high-energy $e^{+} e^{-}$colliders, Nucl. Phys. B 447 (1995) 3 [INSPIRE].

[27] A.G. Akeroyd, Nonminimal neutral Higgs bosons at LEP-2, Phys. Lett. B 377 (1996) 95 [hep-ph/9603445] [INSPIRE].

[28] P. Ko, Y. Omura and C. Yu, A resolution of the flavor problem of two Higgs doublet models with an extra $\mathrm{U}(1)_{H}$ symmetry for Higgs flavor, Phys. Lett. B 717 (2012) 202 [arXiv: 1204.4588] [INSPIRE].

[29] P. Ko, Y. Omura and C. Yu, Higgs phenomenology in Type-I 2HDM with $\mathrm{U}(1)_{H}$ Higgs gauge symmetry, JHEP 01 (2014) 016 [arXiv:1309.7156] [INSPIRE]. 
[30] P. Ko, Y. Omura and C. Yu, Dark matter and dark force in the type-I inert 2HDM with local $\mathrm{U}(1)_{H}$ gauge symmetry, JHEP 11 (2014) 054 [arXiv: 1405.2138] [INSPIRE].

[31] P. Ko, Y. Omura and C. Yu, Higgs and dark matter physics in the type-II two-Higgs-doublet model inspired by $E_{6}$ GUT, JHEP 06 (2015) 034 [arXiv:1502.00262] [INSPIRE].

[32] A. Pich and P. Tuzon, Yukawa alignment in the two-Higgs-doublet model, Phys. Rev. D 80 (2009) 091702 [arXiv:0908.1554] [INSPIRE].

[33] P.M. Ferreira, L. Lavoura and J.P. Silva, Renormalization-group constraints on Yukawa alignment in multi-Higgs-doublet models, Phys. Lett. B 688 (2010) 341 [arXiv:1001.2561] [INSPIRE].

[34] S.L. Glashow and S. Weinberg, Natural conservation laws for neutral currents, Phys. Rev. D 15 (1977) 1958 [inSPIRE].

[35] E.A. Paschos, Diagonal neutral currents, Phys. Rev. D 15 (1977) 1966 [inSPIRE].

[36] R.N. Mohapatra and J.C. Pati, Left-right gauge symmetry and an isoconjugate model of CP-violation, Phys. Rev. D 11 (1975) 566 [InSPIRE].

[37] R.N. Mohapatra and J.C. Pati, A natural left-right symmetry, Phys. Rev. D 11 (1975) 2558 [INSPIRE].

[38] G. Senjanović and R.N. Mohapatra, Exact left-right symmetry and spontaneous violation of parity, Phys. Rev. D 12 (1975) 1502 [InSPIRE].

[39] R.N. Mohapatra and G. Senjanović, Neutrino mass and spontaneous parity violation, Phys. Rev. Lett. 44 (1980) 912 [INSPIRE].

[40] R.N. Mohapatra and G. Senjanović, Neutrino masses and mixings in gauge models with spontaneous parity violation, Phys. Rev. D 23 (1981) 165 [inSPIRE].

[41] Z. Chacko, H.-S. Goh and R. Harnik, The twin Higgs: natural electroweak breaking from mirror symmetry, Phys. Rev. Lett. 96 (2006) 231802 [hep-ph/0506256] [INSPIRE].

[42] Z. Chacko, H.-S. Goh and R. Harnik, A twin Higgs model from left-right symmetry, JHEP 01 (2006) 108 [hep-ph/0512088] [INSPIRE].

[43] J.L. Diaz-Cruz and E. Ma, Neutral SU(2) gauge extension of the standard model and a vector-boson dark-matter candidate, Phys. Lett. B 695 (2011) 264 [arXiv:1007.2631] [INSPIRE].

[44] S. Bhattacharya, J.L. Diaz-Cruz, E. Ma and D. Wegman, Dark vector-gauge-boson model, Phys. Rev. D 85 (2012) 055008 [arXiv:1107. 2093] [inSPIRE].

[45] S. Fraser, E. Ma and M. Zakeri, SU(2) $)_{N}$ model of vector dark matter with a leptonic connection, Int. J. Mod. Phys. A 30 (2015) 1550018 [arXiv:1409.1162] [InSPIRE].

[46] P.Q. Hung, A model of electroweak-scale right-handed neutrino mass, Phys. Lett. B 649 (2007) 275 [hep-ph/0612004] [INSPIRE].

[47] L. Randall and R. Sundrum, A large mass hierarchy from a small extra dimension, Phys. Rev. Lett. 83 (1999) 3370 [hep-ph/9905221] [INSPIRE].

[48] L. Randall and R. Sundrum, An Alternative to compactification, Phys. Rev. Lett. 83 (1999) 4690 [hep-th/9906064] [INSPIRE].

[49] L. Álvarez-Gaumé and E. Witten, Gravitational anomalies, Nucl. Phys. B 234 (1984) 269 [INSPIRE]. 
[50] E. Witten, An SU(2) anomaly, Phys. Lett. B 117 (1982) 324 [INSPIRE].

[51] B. Körs and P. Nath, A Stueckelberg extension of the standard model, Phys. Lett. B 586 (2004) 366 [hep-ph/0402047] [INSPIRE].

[52] B. Körs and P. Nath, Aspects of the Stueckelberg extension, JHEP 07 (2005) 069 [hep-ph/0503208] [INSPIRE].

[53] D. Feldman, Z. Liu and P. Nath, Probing a very narrow Z-prime boson with CDF and DO data, Phys. Rev. Lett. 97 (2006) 021801 [hep-ph/0603039] [INSPIRE].

[54] K. Cheung and T.-C. Yuan, Hidden fermion as milli-charged dark matter in Stueckelberg Zprime model, JHEP 03 (2007) 120 [hep-ph/0701107] [INSPIRE].

[55] SlD Electroweak Group, Sld Heavy Flavor Group, Delphi, LEP, AlePh, OPAL, LEP Electroweak Working Group, L3 collaboration, T.S. Electroweak, $A$ combination of preliminary electroweak measurements and constraints on the standard model, hep-ex/0312023 [INSPIRE].

[56] Sld Electroweak Group, Delphi, Aleph, Sld, SLD Heavy Flavour Group, OPAL, LEP Electroweak Working Group, L3 collaboration, S. Schael et al., Precision electroweak measurements on the $Z$ resonance, Phys. Rept. 427 (2006) 257 [hep-ex/0509008] [INSPIRE].

[57] J. Erler, P. Langacker, S. Munir and E. Rojas, Improved constraints on $Z^{\prime}$ bosons from electroweak precision data, JHEP 08 (2009) 017 [arXiv:0906.2435] [INSPIRE].

[58] V.V. Andreev and A.A. Pankov, Constraints on the $Z-Z^{\prime}$ mixing angle from data measured for the process $e^{+} e^{-} \rightarrow W^{+} W^{-}$at the LEP2 collider, Phys. Atom. Nucl. 75 (2012) 76 [INSPIRE].

[59] V.V. Andreev, P. Osland and A.A. Pankov, Precise determination of $Z-Z^{\prime}$ mixing at the CERN LHC, Phys. Rev. D 90 (2014) 055025 [arXiv:1406.6776] [inSPIRE].

[60] CMS collaboration, Search for heavy narrow dilepton resonances in pp collisions at $\sqrt{s}=7$ $T e V$ and $\sqrt{s}=8 \mathrm{TeV}$, Phys. Lett. B 720 (2013) 63 [arXiv:1212.6175] [INSPIRE].

[61] ATLAS collaboration, Search for high-mass dilepton resonances in pp collisions at $\sqrt{s}=8$ TeV with the ATLAS detector, Phys. Rev. D 90 (2014) 052005 [arXiv: 1405.4123] [INSPIRE].

[62] CMS collaboration, Search for physics beyond the standard model in dilepton mass spectra in proton-proton collisions at $\sqrt{s}=8 \mathrm{TeV}$, JHEP 04 (2015) 025 [arXiv:1412.6302] [INSPIRE].

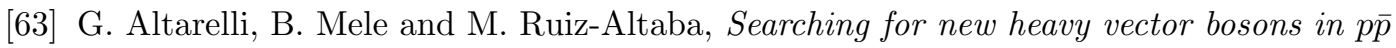
colliders, Z. Phys. C 45 (1989) 109 [Erratum ibid. C 47 (1990) 676] [INSPIRE].

[64] ATLAS collaboration, Measurement of Higgs boson production in the diphoton decay channel in pp collisions at center-of-mass energies of 7 and $8 \mathrm{TeV}$ with the ATLAS detector, Phys. Rev. D 90 (2014) 112015 [arXiv: 1408.7084] [INSPIRE].

[65] CMS Collaboration, Precise determination of the mass of the Higgs boson and studies of the compatibility of its couplings with the standard model, CMS-PAS-HIG-14-009 (2014).

[66] ATLAS collaboration, Search for an invisibly decaying higgs boson produced via vector boson fusion in pp collisions at $\sqrt{s}=8 \mathrm{TeV}$ using the ATLAS detector at the LHC, ATLAS-CONF-2015-004 (2015).

[67] CMS Collaboration, Search for invisible decays of Higgs bosons in the vector boson fusion production mode, CMS-PAS-HIG-14-038 (2014). 
[68] J.F. Gunion, H.E. Haber, G.L. Kane and S. Dawson, The Higgs hunter's guide, Front. Phys. 80 (2000) 1 [INSPIRE].

[69] A. Djouadi, The anatomy of electro-weak symmetry breaking. I: the Higgs boson in the standard model, Phys. Rept. 457 (2008) 1 [hep-ph/0503172] [INSPIRE].

[70] A. Djouadi, The anatomy of electro-weak symmetry breaking. II: the Higgs bosons in the minimal supersymmetric model, Phys. Rept. 459 (2008) 1 [hep-ph/0503173] [INSPIRE].

[71] C.-S. Chen, C.-Q. Geng, D. Huang and L.-H. Tsai, New scalar contributions to $h \rightarrow Z \gamma$, Phys. Rev. D 87 (2013) 075019 [arXiv:1301.4694] [INSPIRE].

[72] A. Pierce and J. Thaler, Natural dark matter from an unnatural Higgs boson and new colored particles at the TeV scale, JHEP 08 (2007) 026 [hep-ph/0703056] [INSPIRE].

[73] G. Bélanger, F. Boudjema, A. Pukhov and A. Semenov, Dark matter direct detection rate in a generic model with MicrOMEGAs 2.2, Comput. Phys. Commun. 180 (2009) 747 [arXiv:0803.2360] [INSPIRE].

[74] G. Bélanger, F. Boudjema, A. Pukhov and A. Semenov, MicrOMEGAs: a tool for dark matter studies, Nuovo Cim. C 033N2 (2010) 111 [arXiv:1005.4133] [INSPIRE].

[75] M.E. Peskin and T. Takeuchi, Estimation of oblique electroweak corrections, Phys. Rev. D 46 (1992) 381 [INSPIRE].

[76] ATLAS collaboration, Search for high-mass diboson resonances with boson-tagged jets in proton-proton collisions at $\sqrt{s}=8 \mathrm{TeV}$ with the ATLAS detector, JHEP 12 (2015) 055 [arXiv: 1506.00962] [INSPIRE]. 